\title{
Small RNAs mediate transgenerational inheritance of genome-wide trans-acting epialleles in maize
}

\author{
Shuai Cao', Longfei Wang ${ }^{1}$, Tongwen Han'1, Wenxue Ye', Yang Liu', Yi Sun², Stephen P. Moose ${ }^{3}$, \\ Qingxin Song ${ }^{1^{*}}$ and Z. Jeffrey Chen ${ }^{4^{*}}$ (D)
}

\author{
*Correspondence: \\ qxsong@njau.edu.cn; \\ zjchen@austin.utexas.edu \\ 1 State Key Laboratory \\ of Crop Genetics \\ and Germplasm \\ Enhancement, Nanjing \\ Agricultural University, \\ 1 Weigang Road \\ Nanjing 210095, China \\ ${ }^{4}$ Department of Molecular \\ Biosciences, The University \\ of Texas at Austin, Austin, TX \\ 78712, USA \\ Full list of author information \\ is available at the end of the \\ article
}

\begin{abstract}
Background: Hybridization and backcrossing are commonly used in animal and plant breeding to induce heritable variation including epigenetic changes such as paramutation. However, the molecular basis for hybrid-induced epigenetic memory remains elusive.

Results: Here, we report that hybridization between the inbred parents B73 and Mo17 induces trans-acting hypermethylation and hypomethylation at thousands of loci; several hundreds $(\sim 3 \%)$ are transmitted through six backcrossing and three selfing generations. Notably, many transgenerational methylation patterns resemble epialleles of the nonrecurrent parent, despite $>99 \%$ of overall genomic loci are converted to the recurrent parent. These epialleles depend on 24-nt siRNAs, which are eliminated in the isogenic hybrid Mo17xB73:mop 1-1 that is defective in siRNA biogenesis. This phenomenon resembles paramutation-like events and occurs in both intraspecific (Mo17xB73) and interspecific (W22xTeosinte) hybrid maize populations. Moreover, siRNA abundance and methylation levels of these epialleles can affect expression of their associated epigenes, many of which are related to stress responses.

Conclusion: Divergent siRNAs between the hybridizing parents can induce transacting epialleles in the hybrids, while the induced epigenetic status is maintained for transgenerational inheritance during backcross and hybrid breeding, which alters epigene expression to enhance growth and adaptation. These genetic and epigenetic principles may apply broadly from plants to animals.
\end{abstract}

Keywords: Hybrid, Small RNA, DNA methylation, Epialleles, Paramutation, Transgenerational inheritance, Backcrossing, Maize

\section{Background}

Transgenerational epigenetic inheritance is the transmission of epigenetic states between generations without alteration of primary DNA sequence [1]. Epigenetic states including DNA methylation and histone modifications may be altered in response to

(c) The Author(s) 2022 Open Access This article is licensed under a Creative Commons Attribution 4.0 International License, which permits use, sharing, adaptation, distribution and reproduction in any medium or format, as long as you give appropriate credit to the original author(s) and the source, provide a link to the Creative Commons licence, and indicate if changes were made. The images or other third party material in this article are included in the article's Creative Commons licence, unless indicated otherwise in a credit line to the material. If material is not included in the article's Creative Commons licence and your intended use is not permitted by statutory regulation or exceeds the permitted use, you will need to obtain permission directly from the copyright holder. To view a copy of this licence, visit http:// creativecommons.org/licenses/by/4.0/. The Creative Commons Public Domain Dedication waiver (http://creativecommons.org/publi cdomain/zero/1.0/) applies to the data made available in this article, unless otherwise stated in a credit line to the data. 
internal (genomic) and external (environmental) signals or stresses in plants and animals $[2,3]$. In animals, toxins and/or nutritional changes can cause alteration of epigenetic states for gene expression, leading to intergenerational variation (not necessary heritable) or transgenerational epigenetic inheritance [3]. Recent studies in Caenorhabditis elegans have shown that small interfering RNAs (siRNAs) are involved in neural gene expression and chemotaxis behavior in three generations [4] and in a long-term memory of avoidance to pathogens [5]. In plants, siRNAs can induce silencing [6] and RNA-directed DNA methylation ( $\mathrm{RdDM}$ ) to enforce epigenetic states [7]. The siRNAmediated RdDM pathway is responsible for stress-induced activation of transposable elements (TEs) in Arabidopsis [8, 9] and rice [10, 11], which involves RdDM for transmission. In rice, stress-induced gene expression is the cause but not the effect of RdDM $[10,11]$.

Allelic interactions in the hybrid (heterozygous state) can induce allelic expression changes in the offspring, as reported in the epigenetic phenomenon known as paramutation in plants [12-15] and later in mice [3], although some paramutation-like events are related to parent-of-origin effects as observed in mice [16], flies [17], and worms [18]. Mechanisms for those trans-acting changes involve small RNAs and RdDM as shown in maize [19]. DNA methylation in plants occurs in CG, $\mathrm{CHH}$, and $\mathrm{CHG}(\mathrm{H}=\mathrm{A}, \mathrm{T}$ or $\mathrm{C})$ [20]; CHH methylation is largely established through the RdDM pathway [21], involving biogenesis of siRNAs by the RNA polymerase Pol II homologs, Pol IV and Pol V, and a RNA-dependent RNA polymerase (RDR) [22, 23]. Paramutation in maize is disrupted in mediator of paramutation1 (mop1), which encodes an RDR2-like protein [19]. In the maize mop 1-1 mutant with loss of function in Mop1-B73, CHH methylation levels are substantially reduced [24], and are accompanied by decrease of 24-nt siRNAs [25]. Moreover, most siRNA distribution differences between the hybridizing parents are also present in the $F_{1}$ progeny [26]. A recent study in the mop 1-1 mutant also found increased recombination in chromosomal arms but reduced recombination in pericentromeric regions [27]. In Arabidopsis thaliana $\mathrm{F}_{1}$ hybrids DNA methylation differences are known as trans-chromosomal methylation (TCM) and demethylation (TCdM) [28]. These TCM and TCdM loci are associated with 24-nt siRNAs, which disappear in $A$. thaliana hybrids of the nrpe1 nrpd1 mutants [29].

Transgenerational inheritance of DNA methylation has been observed in A. thaliana [30]. In maize, differential methylation between inbred lines is heritable, and differentially methylated regions can shift from one epiallele to the other, which are associated with 24-nt siRNAs, and are stably inherited in recombinant inbred lines [31]. In cotton allotetraploids that were formed over $\sim 1.5$ million years ago [32], subsets of hybridization-induced epialleles were maintained during evolution, selection, and domestication, suggesting long-term epigenetic inheritance [33]. However, mechanisms for inheritance of trans-acting epialleles remain poorly understood.

Here, we investigated transgenerational inheritance of genome-wide trans-acting epialleles derived from the reciprocal $\mathrm{F}_{1}$ hybrids ( $\mathrm{BxM}$ and $\mathrm{MxB}$ ) (by convention, the maternal parent is listed first in a genetic cross) between the maize inbred lines B73 (B) and Mo17 (M). The trans-acting differentially methylated regions (DMRs) between $F_{1}$ and two parents were transmitted through six backcrossing followed by three selfing generations; they are called transgenerational DMRs (tgDMRs) or epialleles. Notably, 
many epialleles resembled those from the nonrecurrent parent, despite their genomic sequences were converted to the recurrent parent. Transgenerational inheritance of epialleles was also observed in the interspecific backcross progeny derived from W22 and teosinte. Furthermore, initiation of trans-acting methylation loci in $\mathrm{F}_{1}$ and transmission of epialleles during breeding depended on siRNAs and the RdDM pathway. Finally, some heritable epialleles are associated with expression variation of stress-responsive genes in the $F_{1}$ and backcross-selfing progeny. Collectively, we demonstrate a role of siRNAmediated DNA methylation in genome-wide transgenerational inheritance of epialleles that can influence gene expression and phenotypes in the offspring.

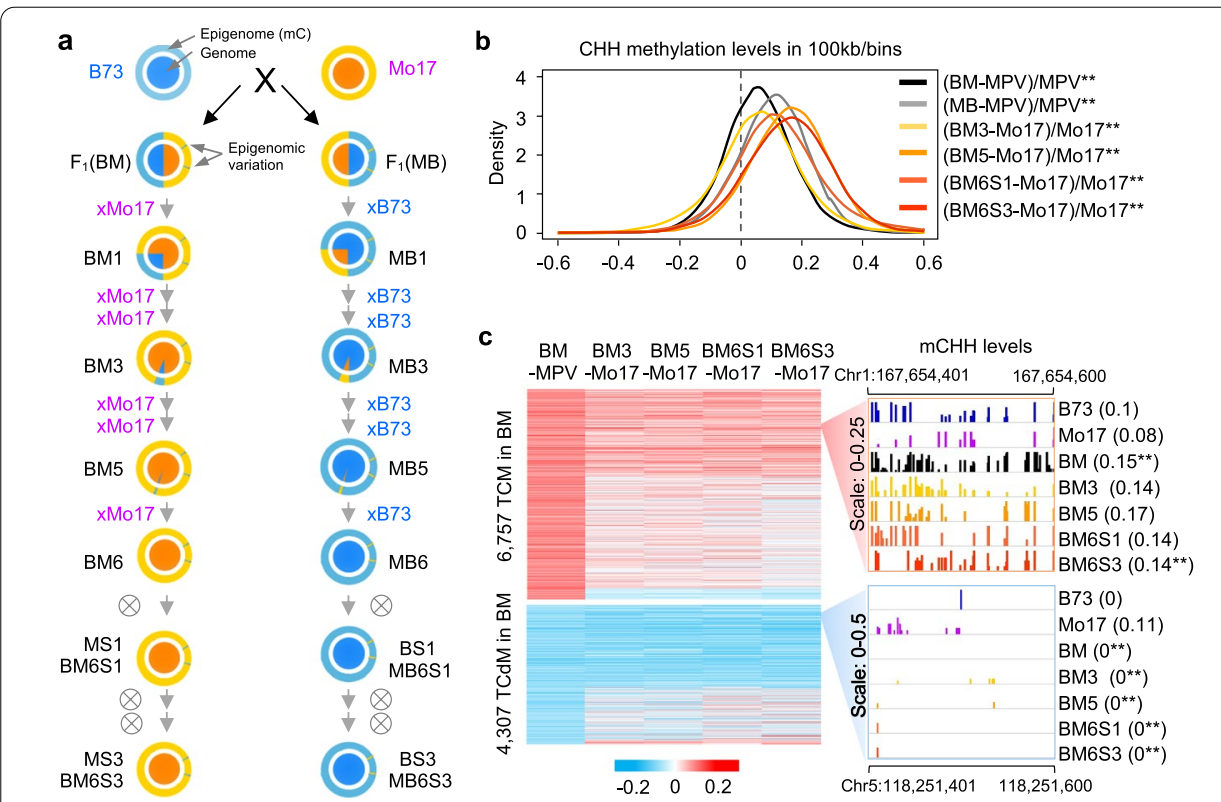

Fig. 1 Hybrid-Induced DNA methylation changes are transgenerational through multiple generations of backcrossing and selfing. a Schemes for producing genetic materials. $F_{1}$ hybrid (BM) was made between B73 (maternal parent) and Mo17 (as pollen donor) and backcrossed consecutively with Mo17 (recurrent parent) to produce backcross lines from BM1 $\left(F_{1} B C 1\right)$ to $B M 5\left(F_{1} B C 5\right)$; BM6 $\left(F_{1} B C 6\right)$ was subsequently self-pollinated as $\mathrm{BM} 6 \mathrm{~S} 1$ for three generations to produce $\mathrm{BM} 6 \mathrm{S3}$. Likewise, the reciprocal $F_{1}$ hybrid $\mathrm{MB}$ underwent a similar backcrossing scheme with $B 73$ pollen to produced $M B 1\left(F_{1} B C 1\right), M B 3\left(F_{1} B C 3\right), M B 5\left(F_{1} B C 5\right)$, and $M B 6$ ( $\left.F_{1} B C 6\right)$ that was self-pollinated to produce MB6S1-MB6S3. Large circles indicate epigenome (methylome) of B73 (light blue) and Mo17 (yellow), and small circles indicate nuclear genomes of B73 (blue) and Mo17 (orange). $\mathbf{b}$ Kernel density distribution of relative methylation changes ( $y$-axis) in the $F_{1}$ hybrids compared to the mid-parent value (MPV) and in the backcross lines compared to their recurrent parents. The density is estimated by the difference (I-II) divided by II (with a 100-kb bin window). Line colors indicate BM (black), MB (gray), BM3 (light yellow), BM5 (yellow), BM6S1 (orange), and BM6S3 (red). Double asterisks indicate statistical significance $(P<0.01$, two-sided Wilcoxon signed-rank test). $\mathbf{c}$ Heatmaps show $\mathrm{CHH}$ methylation changes in the TCM and TCdM loci (left) and two examples (right). Methylation levels were increased in 6757 TCM loci (upper panel) and decreased in 4307 TCdM loci (lower panel) in all backcross and selfing lines examined. Examples are Chr1: 167,654,401-167,654,600 (TCM, upper panel) and Chr5: 118,251,401-118,251,600 (TCdM, lower panel). Numbers in parentheses indicate average methylation levels for the DMRs. Double asterisks indicate statistically significant changes between a $F_{1}$ hybrid and MPV or between a backcross line and its recurrent parent $(P<0.05$, one-way ANOVA test) 


\section{Results}

\section{Methylation changes induced in $\mathrm{F}_{\mathbf{1}}$ hybrids were heritable over multiple generations}

To test epigenetic inheritance, we generated the $F_{1}$ hybrid (Mo17xB73, MB) and the reciprocal hybrid (B73xMo17, BM) (Fig. 1a). The MB hybrid was backcrossed to B73 for six generations (MB1 to MB6), followed by self-pollination for three generations (MB6S1 to MB6S3). Likewise, the reciprocal hybrid (BM) was backcrossed to Mo17 for six generations (BM1 to BM6), followed by self-pollination for three generations (BM6S1 to BM6S3). MB6S3 and BM6S3 populations (55 plants each) were used for genotyping analysis using 20 indel markers (Additional file 1: Fig. S1a). We found that most plants in each population were homozygous for the recurrent parent alleles. Nine of the BM6S3 lines and six of the MB6S3 lines were further genotyped using MaizeSNP6K array [34] (Additional file 2: Table S1). The results confirmed 92.0\% or higher levels of homozygosity to their respective recurrent parents (Additional file 1: Fig. S1b and Additional file 6: Dataset S1a), including BM6S3-2 (99.4\% homozygosity) and MB6S3-3 (99.1\%), which represented BM6S3 and MB6S3, respectively, and were used for further analysis.

Single-base resolution DNA methylomes were generated from 12 genotypes, including inbred parents B73 and Mo17 and their reciprocal $F_{1}$ crosses $(\mathrm{MB}$ and $\mathrm{BM})$, four from 3rd and 5th backcross generations (MB3, MB5, BM3, and BM5), and four reciprocal backcross-selfing lines (BM6S1, BM6S3, MB6S1, and MB6S3) (Additional file 3: Table S2). Sequencing reads of each line with two biological replicates were mapped to the combined genome of B73 and Mo17, and the ratio for the uniquely mapped reads was 1:1 (B73:Mo17) in the $F_{1}$ hybrids (Additional file 1: Fig. S1c and Additional file 6: Dataset S1b). This suggests that these materials are suitable for testing DNA methylation changes during multiple generations of backcrossing and selfing. To avoid excluding duplicate reads, we employed three approaches. Firstly, sequencing reads from B73, MB3, MB5, and MB6S1 and MB6S3 lines were mapped onto the B73 RefGen_v4 genome [35] to detect methylation changes during backcross to B73. Secondly, sequencing reads from Mo17, BM3, BM5, BM6S1, and BM6S3 lines were mapped onto the pseudogenome of Mo17 [36], in which the B73 reference was replaced with corresponding Mo17 SNPs to detect methylation changes among Mo17 backcross lines. Finally, clean reads of reciprocal $\mathrm{F}_{1}$ hybrids (BM and $\mathrm{MB}$ ) were mapped onto B73 RefGen_v4 genome to detect methylation changes induced by hybridization. To minimize the effect of SNPs between B73 and Mo17 (especially between C and T) on DNA methylation analysis, overlapped cytosines between all lines and present in both biological replicates were retained for analysis [33].

These analyses found substantial changes in CHH methylation levels (in 100-kb/bins) in the hybrids or backcross lines (Fig. 1b and Additional file 1: Fig. S1, d-h and Additional file 6: Dataset S1, c-d), while CHG and CG methylation levels did not display obvious changes and could be used as internal controls. For further analysis, we focused on changes in the $\mathrm{CHH}$ methylation. In reciprocal $\mathrm{F}_{1}$ hybrids, $\mathrm{CHH}$ methylation levels were increased relative to the mid-parent value (MPV, average of the two parents) in BM or MB hybrids (Fig. 1b and Additional file 6: Dataset S1e). We tested if the hyper and hypo DMRs induced in the $F_{1}$ hybrids can transmit through backcross breeding. Principal component analysis (PCA) of DMRs showed clear separation between B73 with all backcross lines and Mo17 with its backcross lines, with the reciprocal hybrids (MB and BM) 
in the middle (PC1; Additional file 1: Fig. S2a), suggesting potential inheritance of DMR patterns in backcross generations. As expected, overall methylation changes were positively correlated between $\mathrm{BM} \mathrm{F}_{1}$ hybrid and Mo17 backcross lines (Pearson's correlation coefficient: $\left.r=0.2-0.3, P<2.2 \mathrm{e}^{-16}\right)$ or between $\mathrm{MB} \mathrm{F}_{1}$ hybrid and B73 backcross lines $\left(r=0.25-0.3, P<2.2 \mathrm{e}^{-16}\right)$ (Additional file 1: Fig. S2b and Additional file 7: Dataset S2a). Percentage of variation was higher in the former than in the latter (Additional file 1: Fig. S2a), suggesting a parent-of-origin effect on methylation inheritance, as observed in $A$. thaliana hybrids [37].

We adopted the terms TCM and TCdM to describe trans-acting (chromosomal) methylation (TCM or hyper) and demethylation (TCdM or hypo) events [28], respectively, in which the methylation level of one parental allele in the $\mathrm{F}_{1}$ progeny is trans-altered to resemble the methylation status of the other parental allele. We identified 6757 TCM DMRs and 4037 TCdM DMRs in the BM hybrid and 8723 TCM DMRs and 5212 TCdM DMRs in the reciprocal MB hybrid (Additional file 1: Fig. S2c and Additional file 7: Dataset S2b). The results of more TCM DMRs than TCdM DMRs were consistent with increased overall methylation levels in both $\mathrm{MB}$ and BM hybrids (Fig. 1b and Additional file 6: Dataset S1e). Only a small proportion of TCM DMR (704) and TCdM DMR (652) loci was shared between the two hybrids ( $P=0$ in TCM or TCdM DMRs, hypergeometric test) (Additional file 1: Fig. S2e), suggesting methylation increase or decrease of these alleles in both hybrids, while the majority of them exhibit parent-of-origin effects.

We tabulated numbers of DMRs in all generations including $\mathrm{F}_{1}$ (TCdM and TCM), BM5 and MB5, MB6S1 and BM6S1, and MB6S3 and BM6S3 populations tested (Table 1). Approximately 7.5\% TCdM (hypo) DMRs and 2.4\% TCM (hyper) DMRs in the BM hybrid were also identified as hypo DMRs (Additional file 1: Fig. S3b and Additional file 7: Dataset S2d) and hyper DMRs (Additional file 1: Fig. S3c), respectively, in each of Mo17 backcross generation (BM6S1 or BM6S3). The same trend was observed in the B73 backcross lines, including 11\% TCdM DMRs (Additional file 1: Fig. S3d and Additional file 7: Dataset S2e) and 2.8\% TCM DMRs (Additional file 1: Fig. S3e) in the MB hybrid, which remained hypo- and hypermethylation in respective $B 73$ backcross generation (MB6S1 or MB6S3). As examples, 102 TCM (hyper) DMRs in the BM hybrid remained as hyper DMRs in BM6S3 and also showed increased methylation levels in all

Table 1 Hybridization-induced CHH DMRs and their transgenerational (tgDMR) inheritance in maize

\begin{tabular}{|c|c|c|c|c|c|c|c|c|}
\hline \multirow[t]{2}{*}{ DMRs (in $F_{1}$ ) } & \multirow[t]{2}{*}{$F_{1}(B M)$} & \multicolumn{4}{|c|}{$\begin{array}{l}\text { No. of TCM or TCdM DMRs } \\
\text { present in each Mo17 back- } \\
\text { cross line }\end{array}$} & \multirow{2}{*}{$\begin{array}{l}\text { tgDMRs } \\
\text { present } \\
\text { in BM6S3 } \\
\text { line }\end{array}$} & \multirow[t]{2}{*}{$\begin{array}{l}\text { tgDMRs with } \\
\text { SNPs }\end{array}$} & \multirow{2}{*}{$\begin{array}{l}\text { tgDMRs with } \\
\text { methylation levels } \\
\text { similar to that of } \\
\text { the nonrecurrent } \\
\text { parent Mo17 }\end{array}$} \\
\hline & & ВМз & BM5 & BM6S1 & BM6S3 & & & \\
\hline Hyper (TCM) & 8417 & 218 & 270 & 271 & 188 & 102 & 19 & 2 \\
\hline Hypo (TCdM) & 3561 & 388 & 301 & 241 & 307 & 225 & 60 & 116 \\
\hline \multirow[t]{2}{*}{ DMRs (in $F_{1}$ ) } & $F_{1}(M B)$ & \multicolumn{4}{|c|}{$\begin{array}{l}\text { No. of TCM or TCdM DMRs } \\
\text { present in each B73 back- } \\
\text { cross line }\end{array}$} & \multirow{2}{*}{$\begin{array}{l}\text { tgDMRs } \\
\text { present } \\
\text { in MB6S3 } \\
\text { line }\end{array}$} & \multirow[t]{2}{*}{$\begin{array}{l}\text { tgDMRs with } \\
\text { SNPs }\end{array}$} & \multirow{2}{*}{$\begin{array}{l}\text { tgDMRs with } \\
\text { methylation levels } \\
\text { similar to that of } \\
\text { the nonrecurrent } \\
\text { parent B73 }\end{array}$} \\
\hline & & MB3 & MB5 & MB6S1 & MB6S3 & & & \\
\hline Hyper (TCM) & 6757 & 269 & 180 & 274 & 221 & 99 & 19 & 27 \\
\hline Hypo (TCdM) & 4037 & 433 & 497 & 432 & 456 & 363 & 35 & 155 \\
\hline
\end{tabular}


other Mo17 backcross generations (Table 1), while 225 TCdM (hypo) DMRs remained as hypo DMRs in BM6S3 with decreased methylation levels in all other backcross lines. These TCM and TCdM DMRs that were induced by hybridization and transmitted to the last backcross-selfing generation (BM6S3 or MB6S3) were called transgenerational DMRs (tgDMRs) or epialleles. For example, the TCM region (Chr1: 167,654,401167,654,600) in BM remained to be methylated in all Mo17 backcross lines tested, including BM3, BM5, BM6S1, and BM6S3 (Fig. 1c), whereas the TCdM region (Chr5: $118,251,401-118,251,600)$ in BM had low methylation levels among all generations of Mo17 backcross lines (Fig. 1c).

Interestingly, although TCM DMRs (6757 in the MB hybrid and 8723 in the BM hybrid) were higher than TCdM DMRs (4037 in the BM hybrid, and 5212 in the MB hybrid) (Additional file 1: Fig. S2c and Additional file 7: Dataset S2b), 3-6-fold or more TCdM DMRs than TCM DMRs (5.5\% vs. $1.5 \%$ in the BM hybrid and $6.9 \%$ vs. $1.1 \%$ in the MB hybrid) were transmitted in the Mo17 and B73 backcross-selfing populations, respectively.

These 327 and 462 tgDMRs in Mo17 and B73 backcross lines, respectively (Table 1), were significantly enriched in $5^{\prime}(12.8 \%$ and $15.3 \%$ in Mo17 and B73 backcross lines, respectively) and $3^{\prime}$ (12.2\% and 7.6\% in Mo17 and B73 backcross lines, respectively) regions flanking the coding sequence (Additional file 1: Fig. S4a and Additional file 7: Dataset S2h) ( $P=0$, chi-square test). To determine a possible effect of structural variation (SV) on tgDMRs between B73 and Mo17 genomes, we identified 175,849 SV (Additional file 1: Fig. S4b and Additional file 7: Dataset S2i) and found that 2.1\% (7/327) tgDMRs in Mo17 backcross-selfing lines and 3.9\% (18/462) tgDMRs in B73 backcross-selfing lines overlapped with SV breakpoints, which was higher than $1.3 \%$ $(58,721 / 4,645,542)$ at the whole genome level (Additional file 1: Fig. S4c and Additional file 7: Dataset S2j) ( $P=0$, chi-square test). Furthermore, we found $2.1 \%(949 / 44,799)$ of all DMRs between B73 and Mo17 overlapped with SV (Additional file 1: Fig. S4d), suggesting a contribution of SV to methylation changes in flanking loci [38]. The tgDMRs with SV (7 in Mo17 backcross-selfing lines and 18 in B73 backcross-selfing lines) showed higher levels of methylation changes than tgDMRs without $\mathrm{SV}(P<1.8 \mathrm{e}-3$, Wilcoxon signed-rank test) (Additional file 1: Fig. S4d). Although the total number of tgDMRs with SV was small, these SV-associated DMRs tended to be transgenerationally inherited.

We further investigated chromatin status of tgDMRs using published epigenetic datasets, including Dnase-seq [39], ATAC-seq, and ChIP-seq of histone modifications (H3K27me3, H3K36me3, H3K56ac, H3K4me3, H3K4me1) [40] (NCBI accession no. PRJNA381532, PRJNA527732). We found that these tgDMRs (327 in BM6S3 and 462 in MB6S3) were associated with reduced chromatin accessibility (Additional file 1: Fig. S4f and Additional file 7: Dataset S2k) and depleted with H3K36me3, H3K56ac, H3K4me3, and H3K4me1 histone marks and H2A.Z (Additional file 1: Fig. S4g and Additional file 7: Dataset S2k). In contrast, H3K27me3, a repressive mark for gene expression, overlapped with these DMRs (Additional file 1: Fig. S4g and Additional file 7: Dataset S2k). These results indicate association of tgDMRs with chromatin inaccessibility and void of open chromatin sites. 


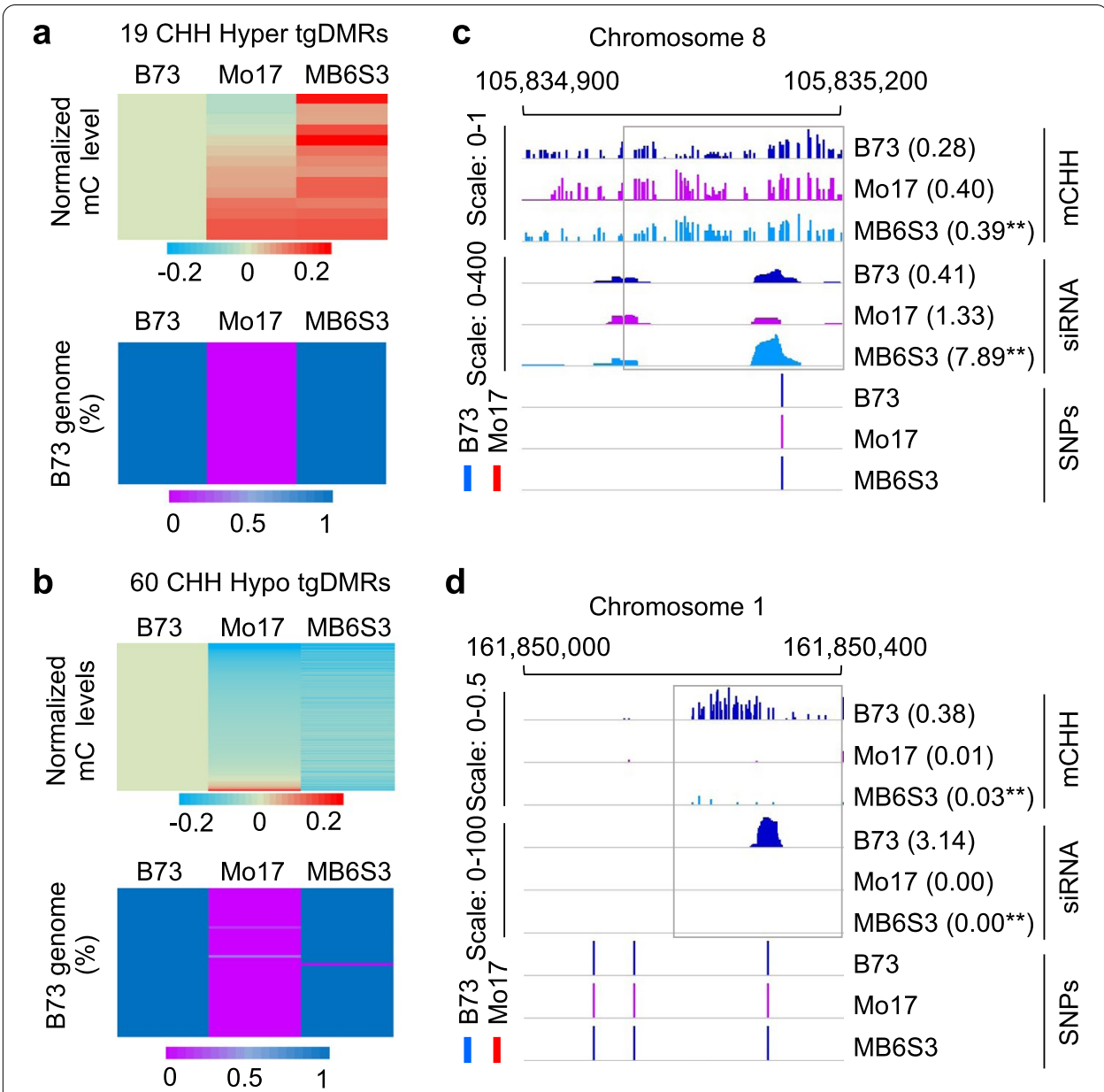

Fig. 2 Transgenerational DNA methylation changes resembled the nonrecurrent parent. $\mathbf{a}, \mathbf{b}$ Inheritance of 19 hyper allelic tgDMRs (a) and 60 hypo allelic tgDMRs (b) in the MB6S3 line (upper panels). Genomic regions in corresponding DMRs were converted to the recurrent parent B73 (lower panels). The percentage was estimated by the average B73/Mo17 allelic ratio in each DMR using SNPs of all loci. c, $\mathbf{d}$ Examples of Chr8: 105,835,001-105,835,200 (c) and Chr1: 161,850,201-161,850,400 (d) displaying high (c) and low (d) methylation and siRNA levels in both MB6S3 and donor parent Mo17. Scales are 0-1 or 0.5 and 0-400 or 100 for normalized methylation and siRNA levels, respectively, in each DMR. Numbers represent average levels of DMR methylation and siRNA expression (RPTM). Double asterisks indicate a significant change between MB6S3 and the recurrent parent B73 $(P<0.05$, one-way ANOVA test for methylation and two-tailed Student's t-test for siRNA expression)

\section{Transgenerational DNA methylation changes resembled the nonrecurrent parent}

Among all tgDMRs in BM6S3 and MB6S3 populations, there were allelic tgDMRs each with SNPs that could discriminate between B73 and Mo17 alleles, including 38 hyper (19 in MB6S3 and 19 in BM6S3) and 95 hypo (60 in MB6S3 and 35 in BM6S3) tgDMRs (Table 1, Fig. 2a, b, and Additional file 1: Fig. S3f, $h$ and Additional file 7: Dataset S2, f-g). Notably, all allelic tgDMRs with one exception were genetically homozygous to the recurrent parent in the last backcross-selfing generation. Only the locus (Chr9:141101601-141101800) had residual B73 sequence in BM6S3.

Inheritance of these tgDMRs may suggest that trans-acting methylation changes in the $F_{1}$ hybrids induced by the nonrecurrent parent were heritable during meiosis. 
As predicted, either hyper or hypo tgDMRs in MB6S3 (backcrossed to B73) resembled the high or low methylation levels of the nonrecurrent parent Mo17 (Fig. 2, a and b, upper panel and Additional file 7: Dataset S2f), despite $>99 \%$ overall genomic fragments of these DMRs in the MB6S3 were converted to the recurrent parent B73 (Fig. 2, a and b, bottom panel). For example, methylation levels of the hyper tgDMR (Chr8: 105,835,001-200) were high in both MB6S3 and Mo17 (Fig. 2c). Likewise, the hypo tgDMR (Chr1: 161,850,201-400) had low methylation levels in both MB6S3 and Mo17 (Fig. 2d). The same trend was observed in the reciprocal BM6S3 line (backcrossed to Mo17) (Additional file 1: Fig. S3f, upper panel and Additional file 1: Fig. S3h, upper panel and Additional file 7: Dataset S2g), which resembled high and low levels of the nonrecurrent parent B73, despite over 99\% genomic regions of these DMRs in the BM6S3 were converted to the recurrent parent Mo17 (Additional file 1: Fig. S3, f and h, bottom panel). For example, the hyper tgDMR (Chr3: 181,013,20118,101,3400) showed high methylation levels in both BM6S3 and B73 (Additional file 1: Fig. S3g), while the hypo tgDMR (Chr1: 227,434,601-227,434,800) displayed low methylation levels in both BM6S3 and B73 (Additional file 1: Fig. S3i). Among tgDMRs in Mo17 and B73 backcross lines (Table 1 and Additional file 4: Table S3), $36.1 \%(118 / 327)$ and $39.0 \%(182 / 462)$ that originated from their corresponding nonrecurrent parents in the $\mathrm{F}_{1}$ were transmitted to the BM6S3 and MB6S3 populations, respectively, after six backcross and three selfing generations (Table 1). This proportion (36-39\%) of tgDMR inheritance was much higher than 1.0\% (44,798 DMRs out of 4,645,542 total genomic bins) in the whole genome level ( $P=0$, chi-square test).

\section{Initiation and maintenance of trans-acting tgDMRs involve 24-nt siRNAs}

This transgenerational inheritance may require trans-acting factor(s) to initiate and maintain these epigenetic states, which can involve siRNAs and RdDM [20, 41]. To dissect these factors, we analyzed distribution of TCM and TCdM DMRs among genomic features and found that they were located more in the $5^{\prime}$ (15\% and 9\% in BM and MB hybrids, respectively) and $3^{\prime}$ (11\% and $6 \%$ in $\mathrm{BM}$ and $\mathrm{MB}$ hybrids, respectively) regions flanking the coding sequence than the genome-wide average (Fig. 3a and Additional file 8: Dataset S3a). The loci in the $5^{\prime}$ region correlated with increased methylation levels (Fig. 3b and Additional file 8: Dataset S3b) $\left(P<7.6 \mathrm{e}^{-19}\right.$ in BM and $P<2.0 \mathrm{e}^{-12}$ in MB, Wilcoxon signed-rank test) and high levels of 24-nt siRNAs (Fig. $3 \mathrm{c}$ and Additional file 8: Dataset S3c) $\left(P<1.6 \mathrm{e}^{-5}\right.$ in BM and $P<3.4 \mathrm{e}^{-29}$ in MB, Wilcoxon signed-rank test). Other siRNAs such as 21/22-nt siRNAs may also be involved [42]. Indeed, distribution patterns of 21/22-nt siRNAs are consistent with those of 24-nt siRNAs (Additional file 1: Fig. S4e and Additional file 7: Dataset S2k), as these noncanonical siRNAs may involve silencing of TEs [42]. Those 24-nt siRNAs were present in 37.1\% (BM) and 34.2\% (MB) of hyper DMRs and 29.6\% (BM) and 33.1\% (MB) of hypo DMRs (Additional file 1: Fig. S5a and Additional file 8: Dataset S3d), which were significantly higher than the genome average (6.8\% in $\mathrm{BM}$ and $6.5 \%$ in $\mathrm{MB})(P<0.05$, Student's $t$-test). These data suggest that 24 -nt siRNAs correlate with trans-acting loci in the reciprocal hybrids.

In maize, $M O P 1$, an ortholog of $R D R 2$, is involved in biogenesis of 24-nt siRNAs and paramutation [19]. We generated and validated the Mo17XB73:mop1-1 hybrid lines to test a role of small RNAs and RdDM in tgDRMs during backcrossing. The reciprocal 


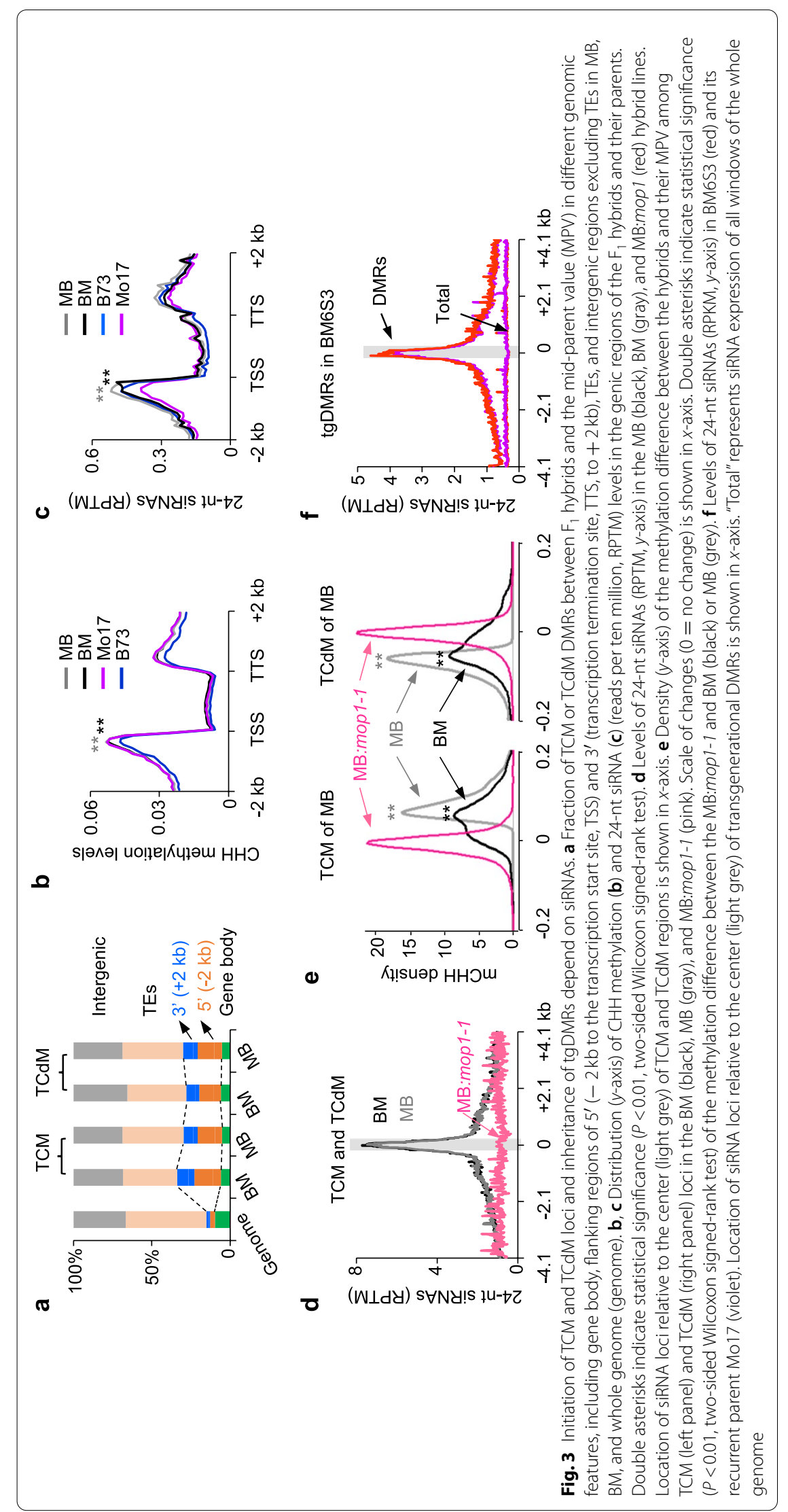


hybrid (B73XMo17:mop1-1) was not used for this study, which might exclude some changes. However, in Arabidopsis, the same set of 24-nt siRNA and $\mathrm{CHH}$ methylation loci was lost in the reciprocal crosses ColXC24:nrpd1/nrpe1 and C24XCol:nrpd1/nrpe1 [29], suggesting similar siRNA and RdDM changes in both directions in vegetative tissues (leaves), except in the seeds, as observed in Arabidopsis [43, 44]. We found that the abundance of 24-nt siRNAs was substantially reduced in the hybrid MB:mop1-1, as in the B73:mop1-1 and Mo17:mop1-1 parents (Additional file 1: Fig. S5, b-d and Additional file 8: Dataset S3e) [26]. Moreover, these 24-nt siRNAs of all corresponding TCM and TCdM loci in the MB hybrid were eliminated in the MB:mop1-1 hybrid (Fig. 3d and Additional file 8: Dataset S3g), suggesting positive association of siRNAs with trans-acting methylation.

We then investigated whether 24-nt siRNAs are required to initiate trans-acting methylation changes in maize $F_{1}$ hybrids. Methylome-seq libraries were made from B73:mop 1-1, Mo17:mop 1-1 and their hybrid MB:mop1-1 each with two biological replicates. Using the same bioinformatic pipeline, we found that methylation levels of CHG and $\mathrm{CHH}$, except for CG, were substantially reduced in all mop1-1 mutants (Additional file 1: Fig. S5, e-g and Additional file 8: Dataset S3f) [27]. Loss of CHH DNA methylation in these mutants occurred in the $5^{\prime}$ and $3^{\prime}$ regions flanking the coding sequence in the MB:mop 1-1 hybrid and its parents (Additional file 1: Fig. S5e and Additional file 8: Dataset S3f), and the methylation levels were evenly reduced in the MB:mop1-1 hybrid (Additional file 1: Fig. S5h and Additional file 8: Dataset S3i) $(P=0$, Wilcoxon signedrank test). Notably, loss of $\mathrm{CHH}$ methylation in the MB:mop1-1 hybrid overlapped with increased $\mathrm{CHH}$ methylation of TCM loci and decreased $\mathrm{CHH}$ methylation of TCdM loci in MB (Fig. 3e and Additional file 8: Dataset S3h) or BM (Additional file 1: Fig. S5i and Additional file 8: Dataset S3j) $\mathrm{F}_{1}$ hybrids $\left(P<5.02 \mathrm{e}^{-44}\right.$, Wilcoxon signed-rank test). These data may indicate that 24-nt siRNAs are required to establish these loci in the hybrids. Alternatively, maintenance of these methylation loci requires 24-nt siRNAs.

The presence of siRNAs in the tgDMRs of BM6S3 and MB6S3 lines (Fig. 2), similar to their respective nonrecurrent parents, suggests that these siRNAs are involved in maintaining these loci during backcross and selfing. To test this, we analyzed 24-nt siRNA abundance and found sharp peaks of the siRNA abundance of tgDMRs in BM6S3 (Fig. 3f and Additional file 8: Dataset S3k) and MB6S3 (Additional file 1: Fig. S5j and Additional file 8: Dataset S3l) lines, relative to the genome average. This suggests persistent presence of these siRNAs in maintaining tgDMRs during backcross and selfing. Moreover, methylation changes in these tgDMRs correlated with loss of siRNAs in the BM:mop1-1 hybrid $\left(P<3.27 \mathrm{e}^{-12}\right.$, Wilcoxon signed-rank test) (Additional file 1: Fig. S5, k-l and Additional file 8: Dataset S3, m-n). It is likely that a low methylation level in the TCdM loci may prevent from transcription by PolIV to generate 24-nt siRNAs. Alternatively, 24-nt siRNAs may dilute in tgDMRs during meiosis through backcrossing and selfing generations. Together, these results indicate that canonical $\mathrm{RdDM}$ pathway establishes TCM and TCdM loci in the $\mathrm{F}_{1}$ hybrids and can maintain them for six backcross and three selfing generations in maize.

This trans-acting methylation occurs not only in the intraspecific hybrids but also in the interspecific maize hybrids that were formed between the modern maize W22 and teosinte (Z. may L. ssp. parviglumis) accessions Bravo (BR) or Blanco (BL) and 

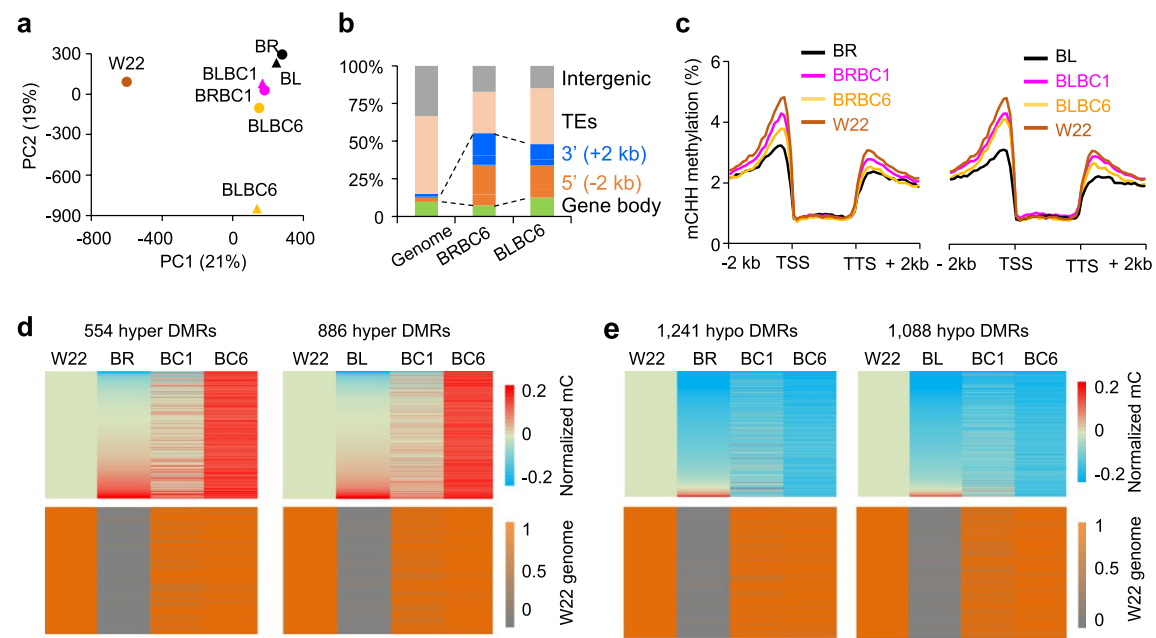

e 1,241 hypo DMRs

1,088 hypo DMRs W22 $\quad$ BR $\quad$ BC1 $\quad$ BC6 $\quad$ W22 $\quad$ BL $\quad$ BC1 $\quad$ BC6
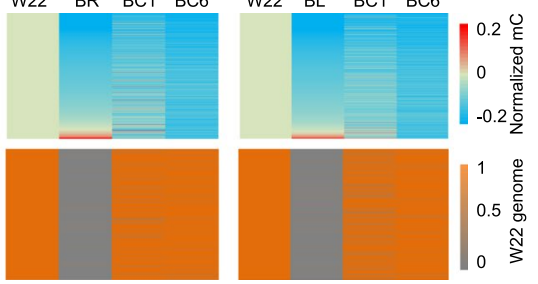

f
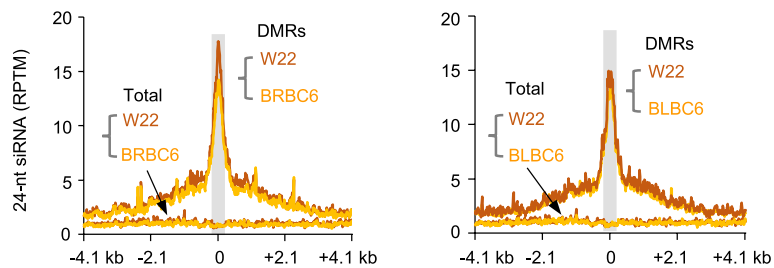

Fig. 4 Transgenerational DNA methylation in backcross lines derived from maize W22 and teosinte. a Principal component analysis (PCA) of $\mathrm{CHH}$ methylation levels with the overall variance explained by PC1 (x-axis) and PC2 (y-axis). Genotypes include W22 (brown dot), BR (Bravo: black dot), BL (Blanco: black triangle), BRBC1 (W22XBR BC1, pink dot), BRBC6 (W22XBR BC6, yellow dot), BLBC1 (W22XBL BC1, pink triangle), and BLBC6 (W22XBL BC6, yellow triangle). b Fraction of CHH DMRs between BRBC (left) or BLBC (right) line and W22 in different genomic features, including gene body (light green) and flanking regions of $5^{\prime}$ (orange, $-2 \mathrm{~kb}$ to transcription start site, TSS) and $3^{\prime}$ (blue, transcription termination site, TTS, to $+2 \mathrm{~kb}$ ), TEs (pink), and intergenic regions (grey) excluding TEs. c Distribution (y-axis) of $\mathrm{CHH}$ methylation in the genic regions of W22 backcross lines, W22, and teosinte. Line colors indicate BR (orange), BRBC1 (pink), BRBC6 (yellow), and W22 (black), left panel, and BL (orange), BLBC1 (pink), BLBC6 (yellow), and W22 (black), right panel. d, e Hyper DMRs (d) of 554 in BRBC6 (left panel) and 866 in BLBC6 (right panel) lines and hypo DMRs (e) of 1241 in BRBC6 (left panel) and 1088 in BLBC6 (right panel) lines. Genomic regions in corresponding DMRs were converted to the recurrent parent W22 (lower panels), which was estimated by the average allelic ratio in each DMR using SNPs of all loci. The methylation level was normalized to that of W22 (0) in each DMR. $\mathbf{f}$ Levels of 24-nt siRNAs (RPTM, $y$-axis) in DMRs between W22 and backcross in W22 (brown), BRBC6 (yellow, left panel), and BLBC6 (yellow, right panel). Location of siRNA loci relative to the center (grey) is shown in $x$-axis. "Total" represents siRNA expression from all windows of the whole genome

transmit to their backcross lines. Using the published data [45], we showed that all backcross lines including BRBC1, BRBC6, BLBC1, and BLBC6 were close to BL and BR parents in PCA plots of methylation levels (Fig. 4a and Additional file 9: Dataset S4a), suggesting that the methylation levels in the backcross lines depend on the nonrecurrent teosinte parents $\mathrm{BR}$ and $\mathrm{BL}$. These trans-acting methylation changes were also enriched in the vicinity of genes (Fig. 4b), especially in the $5^{\prime}$ region flanking the coding sequence (Fig. 4c and Additional file 9: Dataset S4b). Using the DMRs with SNPs that are distinguishable between W22 and teosinte (BR or BL) alleles, we found high methylation levels in most hyper DMRs (Fig. 4d, upper panel and Additional file 9: Dataset S4c) and low methylation level in almost all hypo DMRs (Fig. 4e, upper panel and Additional file 9: Dataset S4d) in the BC6 lines, resembling the 
teosinte parent, while the genome was largely converted to the recurrent parent W22 (Fig. 4, d-e, bottom panel and Additional file 9: Dataset S4, c-d). The data reinforce the notion that trans-acting methylation by the nonrecurrent parent teosinte can be maintained for at least six backcross generations, which also correlated with 24-nt siRNA levels (Fig. 4f and Additional file 9: Dataset S4e). These data together support

a

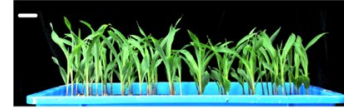

Mo17 BM6S3

b

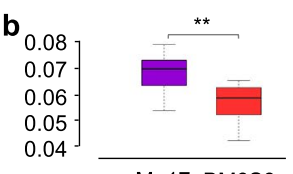

Mo17 BM6S3

c

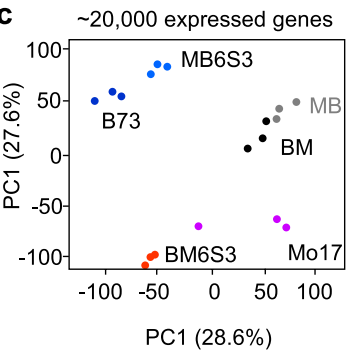

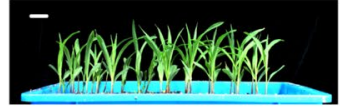
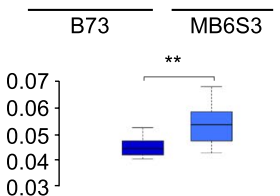

B73 MB6S3

d

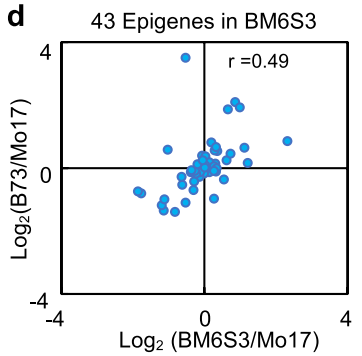

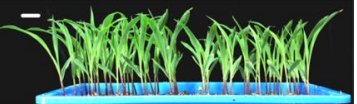

BM

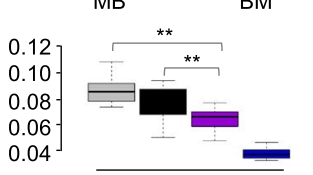

MB BM Mo17 B73

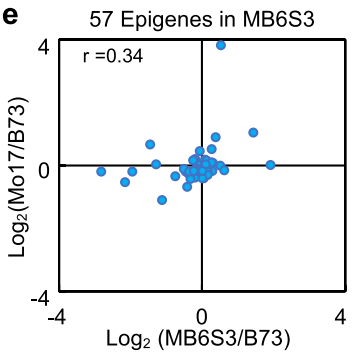

f

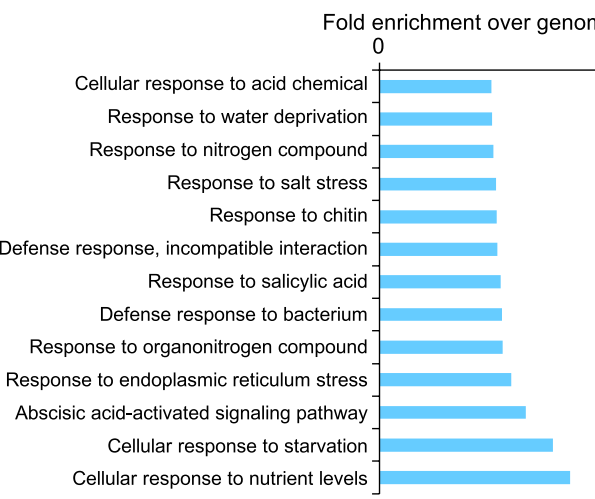

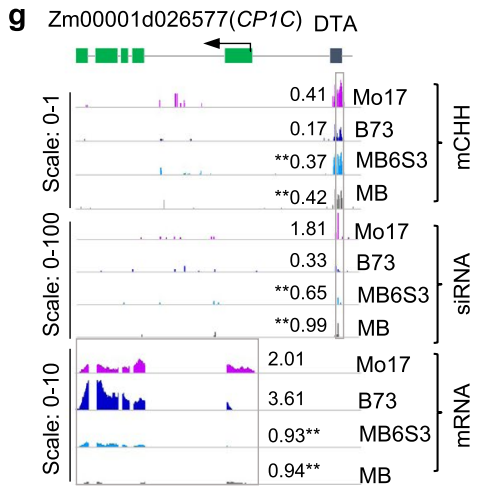

Fig. 5 Transgenerational DMRs correlate with expression levels of epigenes. a, b Seedling phenotypes (a) and aboveground dry weight (b) at the same stage in Mo17 and BM6S3 (left), B73 and MB6S3 (middle), and $\mathrm{MB}$ and $\mathrm{BM}$ hybrids (right). Photos were taken at the same time with the same scale (bars $=10 \mathrm{~mm}$ ). Double asterisks indicate statistical significance $(P<0.05$ and $n=16$ replicates, two-tailed Student's $t$-test). c Principal component analysis (PCA) of transcriptome variation among six lines with the overall variance explained by PC1 ( $x$-axis) and PC2 ( $y$-axis). d Positive correlation ( $r=$ Pearson's correlation coefficient) of expression level changes of 43 epigenes in MB6S3/B73 ( $x$-axis) with those in Mo17/B73 (y-axis). e Positive correlation ( $r=$ Pearson's correlation coefficient) of expression level changes of 57 epigenes in BM6S3/Mo17 ( $x$-axis) with those in in B73/Mo17 ( $y$-axis). f Gene Ontology (GO) overrepresentation of 97 tgDMRs-associated genes in MB6S3 and BM6S3 lines ( $P<0.05$ and fold enrichment $>=2.5$ ). $\mathbf{g}$ An example of the epigene (Zm00001d026577), showing location of DTA transposon, mRNA, siRNA, and CHH methylation levels in Mo17, B73, MB6S3, and MB lines. Scales shown are 0-1 (CHH methylation), 0-100 (siRNA), and 0-10 (mRNA). Numbers indicate average methylation levels of DMR methylation, siRNA expression (RPTM), and gene expression (FPKM). Double asterisks indicate statistically significant changes between MB hybrid and MPV or between MB6S3 and its recurrent parent B73 $(P<0.05$, one-way ANOVA test for methylation; two-tailed Student's $t$-test for siRNA and gene expression) 
a common mechanism for siRNAs in initiation and transgenerational inheritance of trans-acting epialleles induced by inter- and intraspecific crosses in Z. mays.

\section{Roles of trans-acting epialleles of tgDMRs in gene expression and phenotypic variation}

As expected, both BM and MB hybrids exhibited seedling biomass heterosis (Fig. 5, a-b and Additional file 10: Dataset S5a), although grain weight was similar among six genotypes, including B73, Mo17, BM, MB, BM6S3, and MB6S3 (Additional file 1: Fig. S6a and Additional file 10: Dataset S5b). Biomass dry weight was higher in the MB6S3 than in the B73 but lower in BM6S3 than in Mo17 (Fig. 5a, b), despite >99\% overall genomic loci in BM6S3 and MB6S3 lines were converted to their corresponding recurrent parents. This result may suggest a role for these trans-acting DMRs in gene expression and phenotypic variation. Using principal component analysis (PCA) with $\sim 20,000$ expressed genes (EGs), we showed separation of overall gene expression between MB6S3 and B73 and between BM6S3 and Mo17, although MB6S3 and B73 were clearly divergent from BM6S3 and Mo17 (Fig. 5c). This difference of gene expression between the backcrossselfing line and its recurrent parent may suggest an effect of transgenerational methylation changes on gene expression. Here, a gene associated with tgDMR is named an epigene. Among 43 epigenes in BM6S3, $\log _{2}$-fold expression changes in BM6S3/ Mo17 were positively correlated with those in the nonrecurrent parent B73 $(r=0.49$, $P=1.86 \mathrm{e}^{-4}$ ) (Fig. 5d and Additional file 10: Dataset S5e). Moreover, among 57 epigenes in the reciprocal cross MB6S3, $\log _{2}$-fold expression changes in MB6S3/B73 were also positively correlated with those in the nonrecurrent parent $\operatorname{Mo17}\left(r=0.34, P=2.3 \mathrm{e}^{-2}\right)$ (Fig. 5e and Additional file 10: Dataset S5f). At the genome-wide level, we found weak correlation between DNA methylation and expression levels of all epigenes associated with tgDMRs $(P=0.2)$. This is because the correlation between methylation and epigene expression changes may not be straightforward, depending on the genes (housing keeping or otherwise) and location of methylation ( $5^{\prime}$ or $3^{\prime}$ or gene body) $[11,46,47]$. Moreover, when hyper DMRs were related to a repressor such as ROS1, high methylation levels can promote expression of epigenes, as observed for upregulation of expression in sensing methylation homeotsis [48] and in Arabidopsis allotetraploids [49]. There was a trend of elevated expression of epigenes compared to all of genes. Epigenes displayed significantly higher expression fold changes than all genes $\left(P<3.95 \mathrm{e}^{-9}\right.$ in MB6S3 line and $P<1.28 \mathrm{e}^{-8}$ in BM6S3 line, Wilcoxon signed-rank test) (Additional file 1: Fig. S7h and Additional file 10: Dataset S5m). These results suggest that methylation changes in the tgDMRs may alter binding affinity of activators or repressors to alter epigene expression, as recently reported [50]. Interestingly, only three epigenes were shared between MB6S3 and BM6S3 lines, suggesting a strong effect of the nonrecurrent parents on breeding by backcross and selfing.

Notably, the loss of small RNAs in the mop1-1 mutant has a negative effect on seedling growth and biomass heterosis, compared to the wildtype (Additional file 1: Fig. S6, b-c and Additional file 10: Dataset S5c). Moreover, seedling biomass heterosis was also decreased in the hybrid Mo17XB73:mop1-1. The correlation of mop1-1 mutant with the reduced level of seedling growth was consistent with gene expression data. Among five epigenes that were associated with tgDMRs and randomly selected for quantitative RT-PCR analysis (Additional file 5: Table S4), we found that four of five (80\%) epigenes 
tested were significantly upregulated, and the other one (Zm00001d033278) had the same trend of upregulation in the B73:mop1-1 mutant (Additional file 1: Fig. S6d and Additional file 10: Dataset S5d).

Gene ontology (GO) analysis of 97 epigenes in MB6S3 and BM6S3 indicated overrepresentation of biological processes $(P<0.05$ and fold enrichment $>2.5)$ in several stress responsive pathways (Fig. $5 \mathrm{f}$ and Additional file 10: Dataset S5g). Using RNA-seq analysis in each genotype with three biological replicates, we identified differentially expressed genes (DEGs) between the MB6S3 and B73 (3036) and between BM6S3 and Mo17 (3345). Among these DEGs, over $75 \%$ were specific to MB6S3 $(2,274)$ or BM6S3 $(2,583)$, and less than 24\% (762) were shared in both lines (Additional file 1: Fig. S6e), which was consistent with the few overlapping DMRs $(142,<0.1 \%)$ between MB6S3 and BM6S3 (Additional file 1: Fig. S6f).

Approximately $34.5-39.5 \%$ of these tgDMRs were associated with TEs, including class I and II TEs, with no clear enrichment of specific TE families (Additional file 1: Fig. S7, $\mathrm{a}-\mathrm{b}$ and Additional file 10: Dataset S5h). However, these tgDMRs were not enriched among specific TE families (Additional file 1: Fig. S7, a-b) but were associated with longer TEs $(P$ $<1.2 \mathrm{e}^{-4}$, Wilcoxon signed-rank test) (Additional file 1: Fig. S7c and Additional file 10: Dataset S5i), LTRs with older insertion age $\left(P<1.8 \mathrm{e}^{-3}\right.$, Wilcoxon signed-rank test) (Additional file 1: Fig. S7d and Additional file 10: Dataset S5j), and TEs with higher expression levels of 24-nt siRNAs $\left(P<1.4 \mathrm{e}^{-3}\right.$, Wilcoxon signed-rank test) (Additional file 1: Fig. S7e and Additional file 10: Dataset S5i).

These tgDMRs were associated with TEs in their genic regions (Additional file 1: Fig. S7f and Additional file 10: Dataset S5k). Many of stress responsive genes coexist with TEs as observed in Arabidopsis [51] and rice [11] and may regulate expression of TE-associated genes via RdDM pathway [11]. Indeed, among eleven DEGs associated with tgDMRs in BM6S3 line, seven were involved in response to stress, including Zm00001d026577 and Zm00001d017987 (Additional file 1: Fig. S8, a and b). For example, Zm00001d026577 (namely, CP1C) encodes one of cysteine proteases and acts as hubs in plant immunity and in abiotic stress [52]. A short DTA transposon is located in the $5^{\prime}$ region $(-1.5 \mathrm{~kb}$ from TSS) of CP1C (Fig. 5g). In MB6S3 line, DTA was hyper-methylated like the nonrecurrent parent Mo17, consistent with high 24-nt siRNA levels in both MB6S3 and Mo17 lines. Coincidently, CP1C was poorly expressed in Mo17 and nearly silenced in both MB6S3 and MB lines. The majority of 26 genes in response to various stress pathways trended to be down-regulated in two $\mathrm{F}_{1}$ hybrids and MB6S3 (Additional file 1: Fig. S7g and Additional file 10: Dataset S5l). This is reminiscent of a trade-off between downregulation of stressresponsive gene expression and biomass heterosis in A. thaliana intraspecific hybrids [53] and Arabidopsis allotetraploids [54].

These examples show a possible mechanism for siRNAs to maintain trans-acting methylation on a TE-associated tgDMR in the promoter to regulate expression of a stressresponsive gene. Stress-responsive genes are often associated with TEs and tend to show epigenetic regulation and inheritance in Arabidopsis [8, 9]. In maize, these stress-responsive genes may contribute to phenotypic variation such as seedling biomass and adaptation to environmental changes during breeding (Fig. 6). Notably, many paramutation genes are related to TE regulation $[19,55]$ of the genes such as $r 1$, bronze $2(b z 2)$, and $b 1$ loci $[13,14]$ 


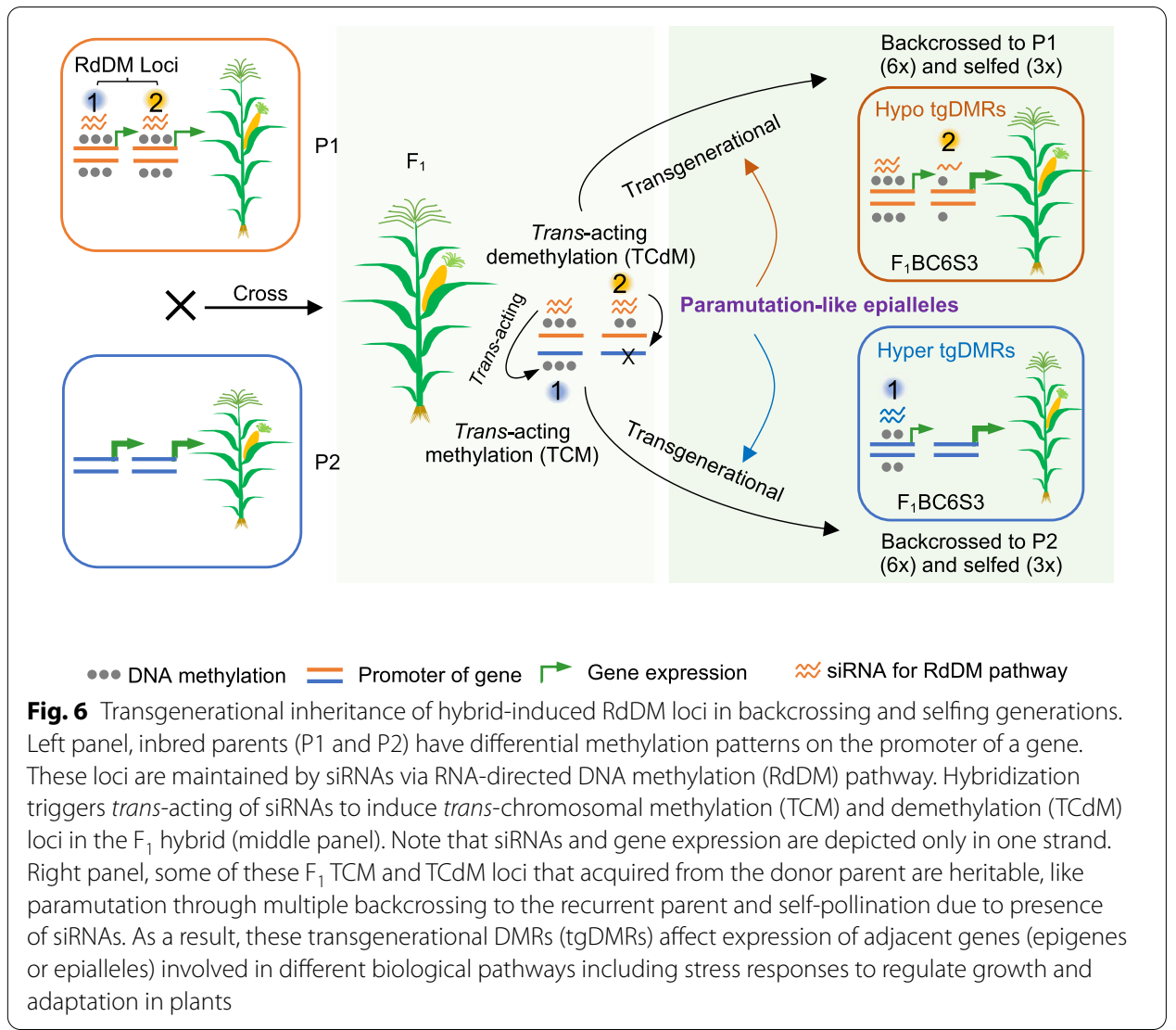

involved in anthocyanin regulatory and biosynthesis pathways. These genes often activate biochemical pathways under the stress, leading to visible color phenotypes.

\section{Discussion}

Biogenesis of 24-nt siRNAs is required for some paramutation events [19], while others involve trans-acting RNA transcripts [56]. In maize hybrids, the divergent population of TE-derived 24-nt siRNAs between the parents may induce RdDM in the hybrids to establish paramutation-like methylation loci, which are maintained during transgenerational inheritance (Fig. 6). These hybrid-induced DMRs are eliminated in the hybrids of mop1-1 mutants in maize, as in the nrpe1/nrpd1 mutant hybrids of Arabidopsis [29]. Thus, RdDM pathway may account for hyper DMRs induced by hybridization. For hypo DMRs, low levels of 24-nt and/or 21/22-nt siRNAs may not sufficiently maintain RdDM $[42,57]$, which may involve other mechanisms such as RdDM-independent pathway $[58,59]$, homeostasis of $\mathrm{CHH}$ methylation and transcriptional regulation of methylated TEs by Pol IV [41], and H3K27me3 histone modifications for non-CG methylation [60]. For example, 37\% of H3K27me3-marked genes are also methylated at non-CG sites in rice [60]. They may form feedback loops, and SDG711 can catalyze H3K27me3 modification via interaction with OsDRM2 for $\mathrm{CHH}$ methylation. 
The involvement of RdDM independent pathway may also explain why more TCdM than TCM loci tend to be transgenerational, since the amount of siRNAs may vary or dilute during selfing and backcross generations, as few siRNAs can transmit from sperm to the zygote in Arabidopsis [43, 61]. Among these trans-acting methylation loci, 5.5-6.9\% hypo-DMR (TCdM) and 1.1-1.5\% hyper-DMR (TCM) loci in the reciprocal hybrids can be transmitted through multiple backcross and selfing generations. This relatively low rate of transgenerational inheritance may result from the homeostasis of siRNAs in TCM loci that have initiated in the $\mathrm{F}_{1}$ hybrid and could be lost during subsequent RdDM process in backcross generations below the threshold levels that are required to maintain the RdDM pathway, as predicted in $A$. thaliana hybrids [62].

In maize recombination inbred lines (RILs), DNA methylation variation in one eighthgeneration RIL switched from non-donor to the donor, which were guided by siRNAs [31]. However, in maize near isogenic lines, DNA methylation variation may correlate with cis-regulatory elements [63], independent of siRNAs. This is probably because the study using DNA methylC immunoprecipitation with microarray profiling may not effectively capture $\mathrm{CHH}$ methylation variation among these lines that are derived from three rounds of backcross.

While traditional paramutation events [12, 14, 64] are independent of cytoplasmic differences, paramutation-like events in mice [16], flies [17], and worms [18] are likely associated with parent-of-origin effects. In this study, B73 happened to possess a similar cytoplasm to Mo17 (Additional file 1: Fig. S8c), which may rule out a possibility of the cytoplasmic effect on establishment of these trans-acting epialleles in reciprocal $F_{1}$ hybrids. However, this notion of the parent-of-origin effect on trans-acting epialleles remains to be investigated.

This mechanism for siRNA-mediated transgenerational inheritance is shared between plants and animals. In C. elegans, small RNAs contribute to transgenerational inheritance of gene expression in neurons for at least three generations via the germline Argonaute HRDE-1 [4]. Similarly, transgenerational inheritance of both gene expression and avoidance behavior to pathogens requires the Piwi Argonaute homolog and its downstream components [5]. It is notable that animals like C. elegans and Drosophila do not have obvious methylation pathways to reinforce epigenetic inheritance. In plants, the siRNA-medicated epigenetic memory is likely reinforced by the RdDM pathway. These data suggest that siRNAs can serve as epigenetic memory in both plants and animals. In plants trans-acting methylation induced by hybridization (internal stress) and cis-acting methylation induced by external stresses [65] can be transgenerational.

Notably, trans-acting DNA methylation changes have a strong effect from the nonrecurrent parent like paramutation on the reciprocal $F_{1}$ hybrids. Over 779 (99.4\%) tgDMRs are specific to the MB6S3 or BM6S3 line (Additional file 4: Table S3). This parent-oforigin effect is likely related to many siRNAs that are maternally transmitted during embryo and endosperm development $[43,44,66]$; these maternally derived siRNAs may exert trans-acting effects on establishing DNA methylation patterns in the hybrids. 


\section{Conclusions}

Transgenerational inheritance of trans-acting DNA methylation epialleles is reminiscent of paramutation in maize [12,67]. Our results suggest that paramutation phenomena are more common than what has been phenotypically recorded. Transgenerational DMRs are observed in both intraspecific (this study) and interspecific [45] hybrids of maize and in the interspecific hybrids and five different allotetraploid cotton species [33]. Because many of these transgenerational DMRs are associated with the genes involved in stress response and domestication related traits [33], the hybridization-induced trans-acting epialleles may serve as a long-term epigenetic memory for adaptation, evolution, and domestication [15], as shown in rice [11]. As paramutation occurs in both maize and mice $[13,14]$, these new insights into transgenerational inheritance of trans-acting epigenetic changes in the plant hybrids may also shed light on epigenetic inheritance in all sexually reproducing organisms including mice and humans.

\section{Methods}

\section{Plant materials}

Maize $\mathrm{F}_{1}$ hybrids and their backcross lines were produced using two inbred lines Mo17 and B73, as illustrated in Fig. 1a. In one direction, B73 was used as the pollen donor to pollinate Mo17 and produce the $\mathrm{F}_{1}$ hybrid MB (Mo17xB73, by convention the maternal parent is listed first in a genetic cross). The $F_{1}(\mathrm{MB})$ hybrid was backcrossed with B73 six times (MB1 to MB6), followed by self-pollination for three generations (MB6S1 to MB6S3). Likewise, the reciprocal $F_{1}$ hybrid (BM, B73xMo17) was backcrossed with Mo17 for six generations (BM1 to BM6), followed by self-pollination for three generations (BM6S1 to BM6S3). Seeds and seedlings from these lines were used for measuring phenotypic traits, in addition to DNA and RNA analyses (see below).

To verify genetic composition, 55 plants each from MB6S3 or BM6S3 population were randomly selected for genotyping analysis using 20 pairs of Indel marker primers (Additional file 5: Table S4), with one marker on the long arm and another on the short arm of each of 10 maize chromosomes. Among them, nine BM6S3 lines and six MB6S3 lines each with the same genotype of 20 markers as the corresponding recurrent parent were further genotyped using MaizeSNP6K array [34]. Finally, BM6S3-2 (with 99.4\% of the Mo17 genome) and MB6S3-3 (99.1\% of the B73 genome) lines were selected to represent their respective populations for further analysis.

Two mop1-1 (Mo17 and B73) mutant lines used in a previous study [26] were genotyped and validated by the PCR (Additional file 5: Table S4), and the homozygous mutants were used to make $F_{1}$ hybrids and for further study.

All lines including B73, Mo17, MB, BM, MB3, MB5, BM6S1, BM6S3, BM3, BM5, MB6S1, MB6S3, B73:mop1-1, Mo17:mop1-1, and MB:mop1-1 (Additional file 3: Table S2) were planted in a walk-in growth chamber with a light/dark cycle of $16 / 8 \mathrm{~h}$ at $28^{\circ} \mathrm{C} / 24^{\circ} \mathrm{C}$. The above ground seeding at 9 days after planting was weighed after dehydration for B73, Mo17, MB, BM, BM6S3, and MB6S3 lines each with sixteen replicates. The second leaf at 9 DAP was collected at $10 \mathrm{am}$ for isolating and purifying DNA (with two biological replicates) and RNA (three biological replicates), respectively. 


\section{Construction of MethyIC-seq and DNA sequencing libraries}

MethylC-library construction and sequencing were performed using with two biological replicates according to a published protocol [33]. In brief, total genomic DNA $(\sim 2 \mu \mathrm{g})$ was fragmented into $200-500$ bp in size by sonication in a Bioruptor (UCD-600TS, Diagenode, Denville, NJ). The fragmented DNA was end-repaired, followed by 3-end adenylation and adapter ligation. An aliquot $(\sim 0.5 \mu \mathrm{g})$ of adapter-ligated DNA fragments was treated with bisulfateusing Zymo EZ DNA Methylation-Gold ${ }^{\mathrm{TM}}$ kit (D5006), followed by 10 cycles of PCR amplification using KAPA HiFi HotStart, which was subject to purification using VAHTS DNA Clean Beads (Vazyme, N411-03). For each genotype, after quality control assessment, two (biological replicate) libraries each a unique bar code were pooled in an equal amount and sequenced across multiple lanes on an Illumina NovaSeq 6000 machine (paired-end $2 \times 150$ bp reads, Novogene).

For DNA sequencing library, an aliquot $(\sim 0.5 \mu \mathrm{g})$ of adapter-ligated DNA (200-500 bp) was amplified by 6 cycles of PCR using Q5 HiFi HotStart and subject to purification using VAHTS DNA Clean Beads (Vazyme, N411-03). After quality control assessment, the libraries each with a unique bar code were sequenced across multiple lanes on an Illumina NovaSeq 6000 machine (paired-end $2 \times 150$ bp reads, Novogene).

\section{Construction of mRNA-seq and small RNA-seq libraries}

For each genotype, mRNA-seq libraries were constructed with three biological replicates. In brief, after DNase treatment, total RNA $(1 \mu \mathrm{g})$ was subject to poly(A) enrichment and then fragmented into $200-500 \mathrm{bp}$ in size. The fragmented mRNA was reverse-transcribed to cDNA using random hexamers, followed by 3-end adenylation and adapter ligation. cDNA was amplified by 10 cycles of PCR using Q5 HiFi HotStart and purified using VAHTS DNA Clean Beads (Vazyme, N411-03). For each genotype, mRNA-seq libraries were constructed with three biological replicates and sequenced by Illumina HiSeq X Ten system (paired-end $2 \times 150$ bp reads, Berrygenomics).

After DNase treatment, small RNA fraction (18-30nt) of total RNA was recovered from a $15 \%$ urea-polyacrylamide gel. The small RNAs were ligated to $5^{\prime}$ and $3^{\prime}$ RNA oligo adapters and performed reverse transcription by amplification (10 cycles). The sRNA-seq libraries were constructed with two biological replicates for each genotype, pooled, and sequenced in one lane on an Illumina HiSeq X Ten sequencer (single-end 50 bp reads, Novogene).

\section{SNPs calling and genotyping}

Raw paired-end sequence reads were filtered into clean data using NGSQCtookit v2.3 (http://www.nipgr.res.in/ngsqctoolkit.html). Clean reads of B73, MB6S3, and Mo17 were mapped onto the genome sequence of B73 [35] (B73_RefGen_v4, https://download. maizegdb.org/Zm-B73-REFERENCE-GRAMENE-4.0) using BWA (v0.7.15), allowing three mismatches allowed per read. Only uniquely mapped paired reads were extracted to new bam files using perl scripts, and the potential PCR duplicates were removed using "Samtools rmdup" of Samtools program (version1.3.1) [68]. The remaining reads were used for further analysis. Consecutive steps using Samtools (v0.1.19, http://samto ols.sourceforge.net/) were applied for calling SNPs between B73 and Mo17 reference 
genomes using the pipelines described previously in rice [69]. SNPs between Mo17 and B73 were used for assessing residual Mo17 genome in MB6S3.

To analyze residual B73 genome in BM6S3, clean reads of BM6S3 and Mo17 were aligned onto the Mo17 genome sequence [36] (Mo17_CAU_V1, https://download.maize gdb.org/Zm-Mo17-REFERENCE-CAU-1.0) to call SNPs between BM6S3 and Mo17. The sequences (100 bp) flanking the B73 SNPs are extracted from Mo17_CAU_V1 and were mapped onto B73_RefGen_v4, and SNPs between BM6S3 and Mo17 in the mapped region flanking $20 \mathrm{bp}$ were called for residual loci of B73 genome in BM6S3.

In another analysis, we resequenced cytoplasmic (mitochondrial and chloroplast) genomes of B73 and Mo17 accessions used in this study. Other re-sequencing data of maize and its relatives were downloaded from DDBJ (https://trace.ddbj.nig.ac.jp accession no. SRP011907) and NCBI (https://www.ncbi.nlm.nih.gov/ accession no. PRJNA528290). Chloroplast genome of B73 (accession no. AY928077.1) was downloaded from NCBI to be assembled by parsing into 200-bp bins with a step size of 20-bp. These re-sequencing data were aligned onto mitochondrial and chloroplast genomes of the reference B73_RefGen_v4 using SNP calling through the Samtools' pipeline [68] as noted above. Heteroplasmic sequences (insertions of cytoplasmic genomes in the nuclear genome) were excluded for the analysis. Remaining SNPs from mitochondrial and chloroplast genomes were used to build neighbor-joining trees using MEGAX with 1000 replicates for bootstrap confidence analysis (Additional file 1: Fig. S8c and Additional file 10: Dataset S5n), while mitochondrial and chloroplast genomes of teosinte (Z. may L. ssp. parviglumis) accessions Bravo (BR) or Blanco (BL) [45] were used as outgroups.

\section{Mapping MethylC-seq reads}

MethylC-seq reads were subject to quality control by NGSQCToolkit_v2.3 (http://www. nipgr.res.in/ngsqctoolkit.html) and then were mapped using Bismark (v0.15.0) with options (-score_min L,0,-0.2 -X 1000) [70]. Bisulphite conversion of all libraries was evaluated using the chloroplast genome (AY928077.1) as a control. The conversion rate ranged from $99.48 \%$ to $99.86 \%$, suggesting a good reproducibility. Clean reads of 12 lines, including B73, MB3, MB5, MB6S1, MB6S3, Mo17, BM3, BM5, BM6S1, and BM6S3, were mapped onto the combine genome sequences of B73 RefGen_v4 and Mo17_CAU_V1. The ratio of uniquely mapped reads onto B73 RefGen_v4 and Mo17_CAU_V1, respectively, in each line was used to evaluate genome composition in a backcross generation.

For detecting methylation changes in backcross lines, we mapped the clean reads of B73, MB3, MB5, MB6S1, and MB6S3 onto the genome sequence of B73_RefGen_v4 [35], while the clean reads from Mo17, BM3, BM5, BM6S1, and BM6S3 were mapped onto the pseudo-Mo17 genome, from which the B73 RefGen_v4 sequences were replaced by corresponding SNPs of Mo17 [36] between Mo17 and B73. For detecting methylation changes induced by hybridization, clean reads from B73, Mo17, MB, BM, B73:mop1-1, Mo17:mop1-1, and MB:mop 1-1 lines were mapped onto the genome sequence of B73 RefGen_v4 [35]. Only the reads mapped to the unique sites covered by at least three reads were retained and used for further analysis. The reads mapped to the same sites were collapsed into a single consensus read to reduce clonal bias. To exclude a mapping bias, the overlapped cytosines among B73, Mo17, BM, and Mo17 backcross lines, 
including BM3, BM5, BM6S1, and BM6S3, were selected for further analysis. The same rule was applied to the overlapped cytosines among B73, Mo17, MB, and B73 backcross lines including MB3, MB5, MB6S1, and MB6S3, as well as among B73, Mo17, MB, BM, B73:mop1-1, Mo17:mop1-1, and MB:mop1-1 lines.

\section{Differentially methylated regions (DMRs) of $\mathrm{CHH}$ context and transgenerational DMRs (tgDMRs)}

CHH DMRs were identified using 200-bp sliding windows. The mean methylation level was calculated for each window [33]. For CHH DMRs, windows containing at least 32 cytosines in the $\mathrm{CHH}$ context covered by at least three reads were selected. DMRs between each comparison (either TCM or TCdM relative to MPV) were determined using an ANOVA test with two biological replications $(P<0.05)$ and cut-off (minimum methylation difference $>0.05$ ).

For TCM and TCdM CHH DMRs in the $\mathrm{F}_{1}$ hybrids to be called as transgenerational DMRs (tgDMRs), they had to meet the two criteria using B73 backcross lines as an example. (1) They are hyper DMRs in MB6S3 compared to B73 and TCM DMRs in the MB hybrid or hypo DMRs in MB6S3 compared to B73 and TCdM DMRs in the MB hybrid. (2) For TCM DMRs in the MB hybrid, methylation level increased in all of other backcross lines including MB3, MB5, and MB6S1 compared to B73; for TCdM DMRs in the MB hybrid, methylation level decreased in all of other backcross lines including MB3, MB5, and MB6S1 compared to B73. The same criteria were applied to the identification of tgDMRs in Mo17 backcross lines.

\section{Analysis of small RNA-seq data}

For small RNA-seq data analysis each with two biological replicates, the raw reads were trimmed from adaptors to retain 18- to 30-nt reads using Cutadapt (v 1.9.1, https:// cutadapt.readthedocs.org/en/stable/) and parsed using the NGSQCToolkit_v2.3 (http:// www.nipgr.res.in/ngsqctoolkit.html) for quality control. Only 18-30-nt long reads were retained and mapped onto B73 RefGen_v4 [35] using Bowtie (v1.1.0) [71], allowing only one unique hit (-m 1) and zero mismatch. After removing the structural RNAs, including rRNA, tRNA, snoRNA, and snRNA fragments, the mapped reads were normalized as reads per ten million (RPTM) [69].

\section{Analysis of mRNA-seq data each with three biological replicates}

As a quality control, all reads were parsed using NGSQCToolkit_v2.3 (http://www.nipgr. res.in/ngsqctoolkit.html). The cleaned RNA-seq reads from 12 libraries (four genotypes including B73, BM, MB, and MB6S3) were mapped onto B73 RefGen_v4 [35]. Cleaned reads from six libraries (Mo17 and BM6S3 lines) were mapped onto the pseudo-Mo17 genome. TopHat soft (v2.1.1) was used for mapping the reads [72]. Uniquely mapped reads were extracted by Perl scripts to determine transcript values by Cufflinks (v2.2.1) [73]. The transcript levels were quantified by FPKM as previously described [69]. Normalized expression values (FPKM) on a $\log 2$ scale were used to evaluate sample relationships by principal component analysis (PCA). Differential expression analysis was 
performed using edgeR [74], and the differentially expressed genes (DEGs) were identified using both the fold-change $(>1.5)$ and analysis of edgeR $(\mathrm{FDR}<0.05)$. Gene Ontology (GO) enrichment analysis was performed using the agriGOv2 Tool (http://syste msbiology.cau.edu.cn/agriGOv2/).

\section{Analysis of transgenerational DMRs and small RNAs between W22 and Teosinte}

The WGBS data, WGS data, and sRNA-seq data published by [45] were downloaded from the National Center for Biotechnology Information (NCBI) with the BioProject accession numbers PRJNA526266, PRJNA528290 and PRJNA528352. The analysis used the reference genome of W22 (https://download.maizegdb.org/Zm-W22-REFERENCENRGENE-2.0) [38] and the bioinformatics' pipelines outlined above.

\section{Analysis of structural variation (SV) and full-length LTRs}

To identify SV, Mo17 genome were divided into 10-kb windows with 100-bp steps (100x depth of genome) and then mapped onto B73 genome (RefGen_v4) using minimap2 software (v2.18-r1015) with default parameters [75]. Mapped results were sorted by Samtools to call SV using cuteSV (v1.0.11) with options "-s 10 -r 500 -1 50 -sl 50" [76]. A total of 175,849 SV were identified, including 94,779 DEL (deletion, 107,516,448 bp of B73-specific sequences, 59.8\%), 77,705 INS (insertion, 62,043,121 bp of Mo17-specific sequences, 34.5\%), 2784 DUP (duplications), 535 INV (inversion), and 46 TRL (translocation). Approximately 2.1\% (7/327) tgDMRs in the Mo17 backcross lines and 3.9\% (18/462) tgDMRs in the B73 backcross lines overlapped with breakpoints of SV, which was higher than $1.3 \%(58,721 / 4,645,542)$ in whole genome.

To investigate insertion age of long-termianl repeats (LTRs), the full-length LTRs of B73 genome (v4) was identified using LTR_FINDER (v1.0.6) [77]. The age was estimated using the mutation rate of $6.5 \times 10^{-9}$ in graases [78].

\section{Analysis of other epigenetic data}

Published epigenetic datasets were downloaded from NCBI (accession no. PRJNA381532, PRJNA527732), including Dnase-seq [39], ATAC-seq, and ChIP-seq of histone modification (H3K27me3, H3K36me3, H3K56ac, H3K4me3, H3K4me1) [40] to investigate chromatin state of tgDMRs. All raw reads were parsed using NGSQCToolkit_v2.3 (http://www.nipgr.res.in/ngsqctoolkit.html), and then clean reads were mapped onto B73 RefGen_v4 [35] using BWA (v0.7.15). Mapped reads with mapping quality of $\geq 10$ were extracted to new bam files, and the potential PCR duplicates were removed using Picard-tools (version 2.0.1) (http://broadinstitute.github.io/picard/). The remaining reads were normalized as reads per million (RPM) for furthe analysis with statistical tests.

\section{Quantitative RT-PCR assays}

To determine the effect of siRNAs on tgDMRs and gene expression, five epigenes each associated with one or more tgDMRs in the B73 backcross lines were randomly selected for analysis. HiScript ${ }^{\circledR}$ II Reverse Transcriptase (RNase H-) (Vazyme, R223-01, Vazyme Biotech Co., Ltd., Nanjing, China) was used to transcribe mRNA into cDNA for qPCR 
assays using a primer pair for each gene (Additional file 5: Table S4) in the reaction of AceQ ${ }^{\circledR}$ qPCR SYBR Green Master Mix (SYBR Green I) (Vazyme, Q111-02).

\section{Supplementary Information}

The online version contains supplementary material available at https://doi.org/10.1186/s13059-022-02614-0.

Additional file 1: Figure S1. Genotyping results and DNA methylation changes in $F_{1}$ hybrids and backcross lines. Figure S2. Hybrid-induced methylation changes were conserved in two $F_{1}$ hybrids and in all backcross lines. Figure S3. Transgenerational DMRs resemble the methylation levels of the donor parent. Figure S4. Genomic features of tgDMRs. Fig. S5. Roles of siRNAs in the initiation and maintenance of TCM and TCdM loci. Figure S6. Correlation between transgenerational DMR and gene expression levels. Figure S7. Characteristics of the TEs associated with tgDMRs. Figure S8. Transgenerational hypermethylated elements targeted by siRNAs in stress-responsive genes.

Additional file 2: Table S1. Nine BM6S3 and six MB6S3 lines were genotyped using MaizeSNP6K.

Additional file 3: Table S2. Summary of high-throughput data for backcrossing lineages.

Additional file 4: Table S3. Transgenerational hybrid-induced DMRs for backcross lines.

Additional file 5: Table S4. Primer pairs used in this study.

Additional file 6: Dataset S1. Hybrid-induced DNA methylation changes are transgenerational in multiple generations of backcrossing and selfing.

Additional file 7: Dataset S2. Transgenerational DNA methylation states resemble the nonrecurrent parent.

Additional file 8: Dataset S3. Initiation and transmission of transgenerational TCM and TCdM loci dependent on siRNAs.

Additional file 9: Dataset S4. Transgenerational DNA methylation in backcross lines derived from modern maize W22 and teosinte.

Additional file 10: Dataset S5. Transgenerational methylation loci correlate with gene expression levels.

Additional file 11. Review history.

\section{Acknowledgements}

We thank Dr. John F. Doebley at University of Wisconsin and Dr. Nathan M. Springer at University of Minnesota for sharing published data of maize-teosinte backcross populations, Dr. Jingya Yuan and Dr. Wu Jiao for helpful discussions, and Dr. Yufeng Wu in the Bioinformatics Center of Nanjing Agricultural University for providing the computing facility for data analysis.

\section{Review history}

The review history is available as Additional file 11.

\section{Peer review information}

Wenjing She was the primary editor of this article and managed its editorial process and peer review in collaboration with the rest of the editorial team.

\section{Authors' contributions}

S.C. and C.Z.J. designed research; S.C., Y.S., L.W., and Y.L. performed research;T.H., Q.S., and W.Y. contributed new reagents and analytical tools; S.C. and Z.J.C. analyzed data; S.P.M provided materials and supervision; S.C. and C.Z.J. wrote the paper. All authors read and approved the paper.

\section{Funding}

The research was supported by grants from the National Natural Science Foundation of China (91631302), Natural Science Foundation of Jiangsu Province (BK20180528), and Jiangsu Collaborative Innovation Center for Modern Crop Production.

\section{Availability of data and materials}

All sequencing data are available under NCBI under the BioProject accession number PRJNA638210 (https://www.ncbi. nlm.nih.gov/bioproject/PRJNA638210) [79]. All datasets generated and/or analyzed in study are available in the article, the source data files that accompany Figs. 1, 2, 3, 4, 5 and 6 and Figs. S1-8, Tables S1, S2, S3 and S4, and Datasets S1, S2, S3, S4 and S5.

Published epigenetic datasets used in this study included Dnase-seq [39], ATAC-seq, and ChIP-seq of histone modifications (H3K27me3, H3K36me3, H3K56ac, H3K4me3, H3K4me1) [40] (NCBl accession no. PRJNA381532, PRJNA527732), and WGBS data, WGS data, and sRNA-seq data [45] (NCBI accession numbers PRJNA526266, PRJNA528290, and PRJNA528352).

\section{Declarations}

Ethic approval and consent to participate Not applicable in this study. 


\section{Competing interests}

The authors declare no competing interests.

\section{Author details}

${ }^{1}$ State Key Laboratory of Crop Genetics and Germplasm Enhancement, Nanjing Agricultural University, 1 Weigang Road, Nanjing 210095, China. ${ }^{2}$ College of Life Sciences, Shanxi Agricultural University, Taigu 030801, China. ${ }^{3}$ Department of Crop Sciences, University of Illinois, Urbana, IL 61801, USA. ${ }^{4}$ Department of Molecular Biosciences, The University of Texas at Austin, Austin, TX 78712, USA.

Received: 19 July 2021 Accepted: 17 January 2022

Published online: 09 February 2022

\section{References}

1. Quadrana L, Colot V. Plant transgenerational epigenetics. Annu Rev Genet. 2016;50:467-91.

2. Heard E, Martienssen RA. Transgenerational epigenetic inheritance: myths and mechanisms. Cell. 2014;157:95-109.

3. Perez MF, Lehner B. Intergenerational and transgenerational epigenetic inheritance in animals. Nat Cell Biol. 2019;21:143-51.

4. Posner R, Toker IA, Antonova O, Star E, Anava S, Azmon E, et al. Neuronal small RNAs control behavior transgenerationally. Cell. 2019;177(1814-1826):e1815.

5. Moore RS, Kaletsky R, Murphy CT. Piwi/PRG-1 argonaute and TGF-beta mediate transgenerational learned pathogenic avoidance. Cell. 2019;177(1827-1841):e1812.

6. Baulcombe DC, Dean C. Epigenetic regulation in plant responses to the environment. Cold Spring Harb Perspect Biol. 2014;6:a019471.

7. Matzke MA, Birchler JA. RNAi-mediated pathways in the nucleus. Nat Rev Genet. 2005;6:24-35.

8. Sanchez DH, Paszkowski J. Heat-induced release of epigenetic silencing reveals the concealed role of an imprinted plant gene. PLoS Genet. 2014;10:e1004806.

9. Ito H, Gaubert H, Bucher E, Mirouze M, Vaillant I, Paszkowski J. An siRNA pathway prevents transgenerational retrotransposition in plants subjected to stress. Nature. 2011;472:115-9.

10. Secco D, Wang C, Shou H, Schultz MD, Chiarenza S, Nussaume L, et al. Stress induced gene expression drives transient DNA methylation changes at adjacent repetitive elements. Elife. 2015;4:e09343.

11. Wang L, Cao S, Wang P, Lu K, Song Q, Zhao FJ, et al. DNA hypomethylation in tetraploid rice potentiates stressresponsive gene expression for salt tolerance. Proc Natl Acad Sci U S A. 2021;118:e2023981118.

12. Brink RA. Paramutation. Annu Rev Genet. 1973;7:129-52.

13. Chandler VL. Paramutation: from maize to mice. Cell. 2007;128:641-5.

14. Hollick JB. Paramutation and related phenomena in diverse species. Nat Rev Genet. 2017;18:5-23.

15. Stam M, Mittelsten Scheid O. Paramutation: an encounter leaving a lasting impression. Trends Plant Sci. 2005;10:283-90.

16. Rassoulzadegan M, Grandjean V, Gounon P, Vincent S, Gillot I, Cuzin F. RNA-mediated non-mendelian inheritance of an epigenetic change in the mouse. Nature. 2006;441:469-74.

17. de Vanssay A, Bouge AL, Boivin A, Hermant C, Teysset L, Delmarre V, et al. Paramutation in Drosophila linked to emergence of a piRNA-producing locus. Nature. 2012;490:112-5.

18. Sapetschnig A, Sarkies P, Lehrbach NJ, Miska EA. Tertiary siRNAs mediate paramutation in C. elegans. PLoS Genet. 2015;11:e1005078.

19. Alleman M, Sidorenko L, McGinnis K, Seshadri V, Dorweiler JE, White J, et al. An RNA-dependent RNA polymerase is required for paramutation in maize. Nature. 2006;442:295-8.

20. Law JA, Jacobsen SE. Establishing, maintaining and modifying DNA methylation patterns in plants and animals. Nat Rev Genet. 2010;11:204-20.

21. Pelissier T, Wassenegger M. A DNA target of $30 \mathrm{bp}$ is sufficient for RNA-directed DNA methylation. RNA. 2000;6:55-65

22. Haag JR, Pikaard CS. Multisubunit RNA polymerases IV and V: purveyors of non-coding RNA for plant gene silencing. Nat Rev Mol Cell Biol. 2011;12:483-92.

23. Herr AJ, Jensen MB, Dalmay T, Baulcombe DC. RNA polymerase IV directs silencing of endogenous DNA. Science. 2005:308:118-20

24. Li Q, Eichten SR, Hermanson PJ, Zaunbrecher VM, Song J, Wendt J, et al. Genetic perturbation of the maize methylome. Plant Cell. 2014;26:4602-16.

25. Nobuta K, Lu C, Shrivastava R, Pillay M, De Paoli E, Accerbi M, et al. Distinct size distribution of endogeneous siRNAs in maize: Evidence from deep sequencing in the mop1-1 mutant. Proc Natl Acad Sci USA. 2008;105:14958-63.

26. Barber WT, Zhang W, Win H, Varala KK, Dorweiler JE, Hudson ME, et al. Repeat associated small RNAs vary among parents and following hybridization in maize. Proc Natl Acad Sci USA. 2012;109:10444-9.

27. Zhao M, Ku JC, Liu B, Yang D, Yin L, Ferrell TJ, et al. The mop1 mutation affects the recombination landscape in maize Proc Natl Acad Sci U S A. 2021;118:e2009475118.

28. Greaves IK, Groszmann M, Ying H, Taylor JM, Peacock WJ, Dennis ES. Trans chromosomal methylation in Arabidopsis hybrids. Proc Natl Acad Sci U S A. 2012;109:3570-5.

29. Zhang Q, Wang D, Lang Z, He L, Yang L, Zeng L, et al. Methylation interactions in Arabidopsis hybrids require RNAdirected DNA methylation and are influenced by genetic variation. Proc Natl Acad Sci U S A. 2016;113:E4248-56.

30. Schmitz RJ, Schultz MD, Lewsey MG, O'Malley RC, Urich MA, Libiger O, et al. Transgenerational epigenetic instability is a source of novel methylation variants. Science. 2011;334:369-73.

31. Regulski M, Lu Z, Kendall J, Donoghue MT, Reinders J, Llaca V, et al. The maize methylome influences mRNA splice sites and reveals widespread paramutation-like switches guided by small RNA. Genome Res. 2013;23:1651-62. 
32. Chen ZJ, Sreedasyam A, Ando A, Song Q, De Santiago LM, Hulse-Kemp AM, et al. Genomic diversifications of five Gossypium allopolyploid species and their impact on cotton improvement. Nat Genet. 2020;52:525-33.

33. Song Q, Zhang T, Stelly DM, Chen ZJ. Epigenomic and functional analyses reveal roles of epialleles in the loss of photoperiod sensitivity during domestication of allotetraploid cottons. Genome Biol. 2017;18:99.

34. Li W, Yang Z, Yao J, Li J, Song W, Yang X. Cellulose synthase-like D1 controls organ size in maize. BMC Plant Biol. 2018;18:239.

35. Jiao Y, Peluso P, Shi J, Liang T, Stitzer MC, Wang B, et al. Improved maize reference genome with single-molecule technologies. Nature. 2017;546:524-7.

36. Sun S, Zhou Y, Chen J, Shi J, Zhao H, Zhao H, et al. Extensive intraspecific gene order and gene structural variations between Mo17 and other maize genomes. Nat Genet. 2018;50:1289-95.

37. Ng DW, Miller M, Yu HH, Huang TY, Kim ED, Lu J, et al. A role for $\mathrm{CHH}$ methylation in the parent-of-origin effect on altered circadian rhythms and biomass heterosis in Arabidopsis intraspecific hybrids. Plant Cell. 2014;26:2430-40.

38. Springer NM, Anderson SN, Andorf CM, Ahern KR, Bai F, Barad O, et al. The maize W22 genome provides a foundation for functional genomics and transposon biology. Nat Genet. 2018;50:1282-8.

39. Burgess SJ, Reyna-Llorens I, Stevenson SR, Singh P, Jaeger K, Hibberd JM. Genome-wide transcription factor binding in leaves from C3 and C4 grasses. Plant Cell. 2019;31:2297-314.

40. Mendieta JP, Marand AP, Ricci WA, Zhang X, Schmitz RJ. Leveraging histone modifications to improve genome annotations. G3 (Bethesda). 2021;11:jkab263.

41. Zhang H, Lang Z, Zhu JK. Dynamics and function of DNA methylation in plants. Nat Rev Mol Cell Biol. 2018:19:489-506.

42. Nuthikattu S, McCue AD, Panda K, Fultz D, DeFraia C, Thomas EN, et al. The initiation of epigenetic silencing of active transposable elements is triggered by RDR6 and 21-22 nucleotide small interfering RNAs. Plant Physiol. 2013;162:116-31.

43. Kirkbride RC, Lu J, Zhang C, Mosher RA, Baulcombe DC, Chen ZJ. Maternal small RNAs mediate spatial-temporal regulation of gene expression, imprinting, and seed development in Arabidopsis. Proc Natl Acad Sci U S A. 2019;116:2761-6.

44. Lu J, Zhang C, Baulcombe DC, Chen ZJ. Maternal siRNAs as regulators of parental genome imbalance and gene expression in endosperm of Arabidopsis seeds. Proc Natl Acad Sci USA. 2012;109:5529-34.

45. Xue W, Anderson SN, Wang X, Yang L, Crisp PA, Li Q, et al. Hybrid decay: a transgenerational epigenetic decline in vigor and viability triggered in backcross populations of teosinte with maize. Genetics. 2019;213:143-60.

46. Zhang X, Yazaki J, Sundaresan A, Cokus S, Chan SW, Chen H, et al. Genome-wide high-resolution mapping and functional analysis of DNA methylation in Arabidopsis. Cell. 2006;126:1189-201.

47. Gent JI, Ellis NA, Guo L, Harkess AE, Yao Y, Zhang X, et al. CHH islands: de novo DNA methylation in near-gene chromatin regulation in maize. Genome Res. 2013;23:628-37.

48. Lei M, Zhang H, Julian R, Tang K, Xie S, Zhu JK. Regulatory link between DNA methylation and active demethylation in Arabidopsis. Proc Natl Acad Sci U S A. 2015;112:3553-7.

49. Jiang $X$, Song $Q$, Ye W, Chen ZJ. Concerted genomic and epigenomic changes accompany stabilization of Arabidopsis allopolyploids. Nat Ecol Evol. 2021;5:1382-93.

50. Han T, Wang F, Song Q, Ye W, Liu T, Wang L, et al. An epigenetic basis of inbreeding depression in maize. Sci Adv. 2021;7:eabg5442.

51. Hollister JD, Gaut BS. Epigenetic silencing of transposable elements: a trade-off between reduced transposition and deleterious effects on neighboring gene expression. Genome Res. 2009;19:1419-28.

52. Schulze Huynck J, Kaschani F, van der Linde K, Ziemann S, Muller AN, Colby T, et al. Proteases underground: analysis of the maize root apoplast identifies organ specific papain-like cysteine protease activity. Front Plant Sci. 2019;10:473.

53. Miller M, Song Q, Shi X, Juenger TE, Chen ZJ. Natural variation in timing of stress-responsive gene expression predicts heterosis in intraspecific hybrids of Arabidopsis. Nat Commun. 2015;6:7453.

54. Wang J, Tian L, Lee HS, Wei NE, Jiang H, Watson B, et al. Genomewide nonadditive gene regulation in Arabidopsis allotetraploids. Genetics. 2006;172:507-17.

55. Chandler VL, Walbot V. DNA modification of a maize transposable element correlates with loss of activity. Proc Natl Acad Sci U S A. 1986;83:1767-71.

56. Arteaga-Vazquez M, Sidorenko L, Rabanal FA, Shrivistava R, Nobuta K, Green PJ, et al. RNA-mediated trans-communication can establish paramutation at the b1 locus in maize. Proc Natl Acad Sci U S A. 2010:107:12986-91.

57. Greaves IK, Eichten SR, Groszmann M, Wang A, Ying H, Peacock WJ, et al. Twenty-four-nucleotide siRNAs produce heritable trans-chromosomal methylation in F1 Arabidopsis hybrids. Proc Natl Acad Sci U S A. 2016;113:E6895-902.

58. Song Q, Guan X, Chen ZJ. Dynamic roles for small RNAs and DNA methylation during ovule and fiber development in allotetraploid cotton. PLoS Genet. 2015;11:e1005724.

59. Zemach A, Kim MY, Hsieh PH, Coleman-Derr D, Eshed-Williams L, Thao K, et al. The Arabidopsis nucleosome remodeler DDM1 allows DNA methyltransferases to access H1-containing heterochromatin. Cell. 2013;153:193-205.

60. Zhou S, Liu X, Zhou C, Zhou Q, Zhao Y, Li G, et al. Cooperation between the H3K27me3 chromatin mark and non-CG methylation in epigenetic regulation. Plant Physiol. 2016;172:1131-41.

61. Walker J, Gao H, Zhang J, Aldridge B, Vickers M, Higgins JD, et al. Sexual-lineage-specific DNA methylation regulates meiosis in Arabidopsis. Nat Genet. 2018;50:130-7.

62. Groszmann M, Greaves IK, Albert N, Fujimoto R, Helliwell CA, Dennis ES, et al. Epigenetics in plants-vernalisation and hybrid vigour. Biochim Biophys Acta. 2011;1809:427-37.

63. Li Q, Eichten SR, Hermanson PJ, Springer NM. Inheritance patterns and stability of DNA methylation variation in maize near-isogenic lines. Genetics. 2014;196:667-76.

64. Hollick JB, Patterson Gl, Coe EH Jr, Cone KC, Chandler VL. Allelic interactions heritably alter the activity of a metastable maize pl allele. Genetics. 1995;141:709-19.

65. Zheng X, Chen L, Xia H, Wei H, Lou Q, Li M, et al. Transgenerational epimutations induced by multi-generation drought imposition mediate rice plant's adaptation to drought condition. Sci Rep. 2017;7:39843. 
66. Mosher RA, Melnyk CW, Kelly KA, Dunn RM, Studholme DJ, Baulcombe DC. Uniparental expression of PollV-dependent siRNAs in developing endosperm of Arabidopsis. Nature. 2009;460:283-6.

67. Chandler VL, Stam M. Chromatin conversations: mechanisms and implications of paramutation. Nat Rev Genet. 2004;5:532-44.

68. Li H, Handsaker B, Wysoker A, Fennell T, Ruan J, Homer N, et al. The sequence alignment/map format and SAMtools. Bioinformatics. 2009;25:2078-9.

69. Yuan J, Chen S, Jiao W, Wang L, Wang L, Ye W, et al. Both maternally and paternally imprinted genes regulate seed development in rice. New Phytol. 2017;216:373-87.

70. Krueger F, Andrews SR. Bismark: a flexible aligner and methylation caller for Bisulfite-Seq applications. Bioinformatics. 2011;27:1571-2.

71. Langmead B, Trapnell C, Pop M, Salzberg SL. Ultrafast and memory-efficient alignment of short DNA sequences to the human genome. Genome Biol. 2009;10:R25.

72. Trapnell C, Pachter L, Salzberg SL. TopHat: discovering splice junctions with RNA-Seq. Bioinformatics. 2009;25:1105-11.

73. Trapnell C, Roberts A, Goff L, Pertea G, Kim D, Kelley DR, et al. Differential gene and transcript expression analysis of RNA-seq experiments with TopHat and Cufflinks. Nature Protocols. 2012;7:562-78.

74. Robinson MD, McCarthy DJ, Smyth GK. edgeR: a Bioconductor package for differential expression analysis of digital gene expression data. Bioinformatics. 2010;26:139-40.

75. Li H. Minimap2: pairwise alignment for nucleotide sequences. Bioinformatics. 2018;34:3094-100.

76. Jiang T, Liu Y, Jiang Y, Li J, Gao Y, Cui Z, et al. Long-read-based human genomic structural variation detection with cuteSV. Genome Biol. 2020;21:189.

77. Xu Z, Wang H. LTR_FINDER: an efficient tool for the prediction of full-length LTR retrotransposons. Nucleic Acids Res. 2007;35:W265-8.

78. Gaut BS, Morton BR, McCaig BC, Clegg MT. Substitution rate comparisons between grasses and palms: synonymous rate differences at the nuclear gene Adh parallel rate differences at the plastid gene rbcL. Proc Natl Acad Sci U S A. 1996;93:10274-9.

79. Cao S, Wang L, Han T, Ye W, Liu Y, Sun Y, Moose SP, Song Q, Chen ZJ. Small RNAs mediate transgenerational inheritance of genome-wide trans-acting epialleles in maize. NCBI PRJNA638210: https://www.ncbi.nlm.nih.gov/biopr oject/?term=PRJNA638210. 2022 (this issue).

\section{Publisher's Note}

Springer Nature remains neutral with regard to jurisdictional claims in published maps and institutional affiliations.

Ready to submit your research? Choose BMC and benefit from:

- fast, convenient online submission

- thorough peer review by experienced researchers in your field

- rapid publication on acceptance

- support for research data, including large and complex data types

- gold Open Access which fosters wider collaboration and increased citations

- maximum visibility for your research: over 100M website views per year

At BMC, research is always in progress.

Learn more biomedcentral.com/submissions 